\title{
Higher order resonance stability of triangular libration points for radiating primaries in ER3BP
}

\author{
A Narayan ${ }^{1 *}$, Nutan Singh ${ }^{2}$ \\ ${ }^{1}$ Department of Mathematics, Bhilai Institute of Technology, Durg, 491001, India \\ ${ }^{2}$ Department of Mathematics, Rungta College of Engg. and Technology \\ *Corresponding author E-mail: ashutoshmaths.narayan@gmail.com
}

\begin{abstract}
The main aim of this paper is to study the existence of resonance and stability of the triangular equilibrium points in the framework of ER3BP when both the attracting bodies are sources of radiation at $\omega_{1}=\omega_{2}, \omega_{1}=2 \omega_{2}, \omega_{1}=3 \omega_{2}$ in both circular and elliptical cases .A practical application of this model could be seen in the case of binary systems ( Achird, Luyten, $\alpha$ Cen- AB, Kruger 60 , Xi Bootis). The study is carried out both analytically and numerically by considering various values of radiation pressures and around binary systems .In both cases (CR3BP and ER3BP) it is found that $\omega_{1}=\omega_{2}$ corresponds to the boundary region of the stability for the system, whereas the other two cases $\omega_{1}=2 \omega_{2}, \omega_{1}=3 \omega_{2}$ correspond to the resonant cases. In order to investigate the stability, the Hamiltonian is normalized up to the fourth order by using linear canonical transformation of variables. Then KAM theorem is applied to investigate the stability for different values of radiation pressures in general and around the binary systems in particular. Finally, simulation technique is applied to study the correlation between radiation pressures and mass ratio in circular case; mass ratio and eccentricity in elliptical case. It is found that all the binary systems considered are stable. Also, it is found that except for some values of the radiation pressure parameters and for $\mu \leq \mu_{c}=0.0385209$; the triangular equilibrium points are stable.
\end{abstract}

Keywords: ER3BP; Hamiltonian Functions; Triangular Libration Points; Resonance; KAM Theory.

\section{Introduction}

The stability of the solar system is a longstanding problem. Threebody stability is fundamental to astrophysical processes on all length and mass scales from planetary systems to clusters of galaxies. Over the centuries, mathematicians and astronomers have spent large amount of energy providing stability theorems for dynamical systems closely related to the solar system. The study of the stability of an elliptical restricted three body problem of a Hamiltonian system is generally performed by using KAM theorem.

The nonlinear stability for resonance as well as for the nonresonance cases of the triangular libration points, taking one of the bodies as radiating was studied by Manju and Choudhary (1985). Kumar and Choudhary (1986) investigated the stability of the triangular libration points for non-resonance as well as resonance case; taking both the bodies as radiating in circular restricted three body problems in presence of the third and fourth order resonance. Bhatnagar (1994) discussed the nonlinear stability of the triangular equilibrium points in circular restricted three bodies, considering bigger primary as a source of radiation. The nonlinear stability of the triangular Lagrangian points, considering the bigger primary as oblate spheroid in circular case was examined by Markellos (1996).

The detailed description and the behavior of equilibrium points in ER3BP were given by Danby (1964), Bennet (1965), Szebehely (1967) Rabe (1970), Markeev (1978), Selaru Cucu-Dumitrescu
(1994), Halan and Rana (2001). The influence of the eccentricity of the orbits of the primaries with or without radiation pressure (s) on the existence and stability of the equilibrium points was studied by Gyorgrey ( 1985), Grebenikov (1964), Kumar and Choudhary (1990), Markeellos (1992), Conxita (1995),Shaoo and Ishwar (2000), Roberts (2002), Floria (2004), Zimvoschikov and Thakai (2004), Ammar (2008), Erdi (2009), Kumar and Ishwar (2011). Singh and Umar (2012a), (2012b), Usha et. al. (2014), Narayan and Singh (2014 a, b, c,).

The present paper deals with the stability of the triangular points in non-resonance condition, considering both the primaries as radiating; satisfying the tions $\beta_{1}+\beta_{2} \leq 1 ; \beta_{1}+\beta_{2} \geq 1$; and $0 \leq \beta_{1}, \beta_{2} \leq 1$; . The study is carried out for both circular case and elliptical case.

This paper has been organized in various sections: section 1 described the introduction Section 2 describes the equations of motion of the problem, section 3 deals with characteristics roots and first order stability of the triangular equilibrium points. The existence of resonance is discussed in section 4 and 5 while Section 6 deals with normalization and higher order stability of the libration points. Finally, section 7 summarizes the discussion and conclusion of the paper. 


\section{Equations of motion}

The differential equations of the motion of the infinitesimal mass in elliptical restricted three body problem under radiating primaries in pulsating system is given as:

[Narayan and Singh (2014a)]

$$
\begin{aligned}
& \bar{x}^{\prime \prime}-2 \bar{y}^{\prime}=\varphi \frac{\partial \Omega}{\partial \bar{x}} ; \\
& \bar{y}^{\prime \prime}+2 \bar{x}^{\prime}=\varphi \frac{\partial \Omega}{\partial \bar{y}} ; \\
& \bar{z}^{\prime \prime}+\bar{z}=\varphi \frac{\partial \Omega}{\partial \bar{z}},
\end{aligned}
$$

where

$$
\begin{gathered}
\Omega=(1-\mu)\left[\frac{1}{2} \bar{r}_{1}^{2}+\frac{\left(1-\beta_{1}\right)}{\bar{r}_{1}}\right]+ \\
\mu\left[\frac{1}{2} \bar{r}_{2}^{2}+\frac{\left(1-\beta_{2}\right)}{\bar{r}_{2}}\right]-\frac{1}{2} \frac{z^{2}}{r} ;
\end{gathered}
$$

$\phi=r=(1+e \cos \mathrm{f})^{-1}$

$\bar{r}_{1}^{2}=(\bar{x}+\mu)^{2}+\bar{y}^{2}+\bar{z}^{2}$

$\bar{r}_{2}^{2}=(\bar{x}+\mu-1)^{2}+\bar{y}^{2}+\bar{z}^{2}$.

where $\beta_{1}, \beta_{2}$ is the radiation pressure and $f$ is a true anomaly of the primaries.

The coordinates of the triangular equilibrium points $\mathrm{L}_{4}$ and $\mathrm{L}_{5}$ in $(\bar{x}, \bar{y})$ plane are given by: [Narayan and Singh (2014)]

$\bar{x}=\frac{1}{2}-\mu+\frac{\beta_{2}}{3}-\frac{\beta_{1}}{3}$,

$\bar{y}= \pm \frac{\sqrt{3}}{2}\left[1-\frac{2 \beta_{1}}{9}-\frac{2 \beta_{2}}{9}\right]$

\section{Characteristics roots and first order stabil- ity of the triangular equilibrium points}

It may be easily verified that the system (2.1) describes the motion of dynamical system with Lagrangian given by:

$$
\begin{aligned}
& L=\frac{(\dot{\bar{x}})^{2}+(\dot{\bar{y}})^{2}}{2}+(\dot{\bar{y}} \bar{x}-\dot{\bar{x}} \bar{y})+\frac{1}{1+e \cos f} \times \\
& {\left[(1-\mu)\left(\frac{\bar{r}_{1}^{2}}{2}+\frac{\left(1-\beta_{1}\right)}{\bar{r}_{1}}\right)+\mu\left(\frac{\bar{r}_{2}^{2}}{2}+\frac{\left(1-\beta_{2}\right)}{\bar{r}_{2}}\right)\right]}
\end{aligned}
$$

where

$p_{\bar{x}}=\frac{\partial L}{\partial \dot{\bar{x}}}=\dot{\bar{x}}-\bar{y} ; \quad p_{\bar{y}}=\frac{\partial L}{\partial \dot{\bar{y}}}=\dot{\bar{y}}+\bar{x}$.

Now, the expression for the Hamiltonian function of the problem is constructed using the formula given as follows:
$H=-L+\frac{\partial L}{\partial \dot{\bar{x}}} \dot{\bar{x}}+\frac{\partial L}{\partial \dot{\bar{y}}} \dot{\bar{y}}$

$=-L+p_{\bar{x}} \dot{\bar{x}}+p_{\bar{y}} \dot{\bar{y}}$

$=-L+p_{\bar{x}}\left(p_{\bar{x}}+\bar{y}\right)+p_{\bar{y}}\left(p_{\bar{y}}-\bar{x}\right) ;$

$=-L+\left(p_{\bar{x}}{ }^{2}+p_{\bar{y}}{ }^{2}\right)+\left(p_{\bar{x}} \bar{y}-p_{\bar{y}} \bar{x}\right)$

From equation (3.1) and (3.2), the perturbed Hamiltonian function of the problem is given by:

$$
\begin{aligned}
& H=-\frac{(\dot{\bar{x}})^{2}+(\dot{\bar{y}})^{2}}{2}-(\dot{\bar{y}} \bar{x}-\dot{\bar{x}} \bar{y})-\frac{1}{1+e \cos f} \times \\
& {\left[(1-\mu)\left(\frac{\bar{r}_{1}^{2}}{2}+\frac{\left(1-\beta_{1}\right)}{\bar{r}_{1}}\right)+\mu\left(\frac{\bar{r}_{2}^{2}}{2}+\frac{\left(1-\beta_{2}\right)}{\bar{r}_{2}}\right)\right]} \\
& +\left(p_{\bar{x}}^{2}+p_{\bar{y}}^{2}\right)+\left(p_{\bar{x}} \bar{y}-p_{\bar{y}} \bar{x}\right)
\end{aligned}
$$

After further simplification the perturbed Hamiltonian function in the model of ER3BP considering the photogravitational effect of both the primaries having radiation pressure $\beta_{1}$ and $\beta_{2}$ is given as:

$$
\begin{aligned}
H= & \frac{\left(p_{\bar{x}}^{2}+p_{\bar{y}}^{2}\right)}{2}+\left(p_{\bar{x}} \bar{y}-p_{\bar{y}} \bar{x}\right)+ \\
& \frac{(\bar{x})^{2}+(\bar{y})^{2}}{2}-\frac{1}{1+e \cos f} \times \\
& {\left[(1-\mu)\left(\frac{\bar{r}_{1}^{2}}{2}+\frac{\left(1-\beta_{1}\right)}{\bar{r}_{1}}\right)\right.} \\
& \left.+\mu\left(\frac{\bar{r}_{2}^{2}}{2}+\frac{\left(1-\beta_{2}\right)}{\bar{r}_{2}}\right)\right]
\end{aligned}
$$

Since the two triangular points are symmetrical, the nature of the motion near the two points is the same. So, the motion of the infinitesimal near one of the equilibrium points is considered. For this, we shift the origin at $\mathrm{L}_{4}$. Let us use the change of the variables given by:

$$
\begin{aligned}
& \bar{x}=\xi+q_{1} ; \quad \bar{y}=\eta+q_{2} ; \\
& p_{\bar{x}}=p_{\xi}+p_{1} ; p_{\bar{y}}=p_{\eta}+p_{2}
\end{aligned}
$$

Where the displacement at and near $L_{4}$ is represented as follows:

$\xi=\frac{1}{2}-\mu+\frac{\beta_{2}}{3}-\frac{\beta_{1}}{3}$

$\eta=\frac{\sqrt{3}}{2}\left[1-\frac{2 \beta_{1}}{9}-\frac{2 \beta_{2}}{9}\right]$

$p_{\xi}=-\frac{\sqrt{3}}{2}\left[1-\frac{2 \beta_{1}}{9}-\frac{2 \beta_{2}}{9}\right]$

$p_{\eta}=\frac{1}{2}-\mu+\frac{\beta_{2}}{3}-\frac{\beta_{1}}{3}$

The solutions for equation (3.6) in the new variables are given by the equilibrium position:

$q_{1}=q_{2}=p_{1}=p_{2}=0$

Now expanding the Hamiltonian function given by equation (3.4) in the powers of $\mathrm{p}_{\mathrm{i}}$ and $\mathrm{q}_{\mathrm{i}}$ as: 


$$
\begin{aligned}
H=\sum_{K=0}^{\infty} H_{K} & =H_{0}+H_{1}+H_{2}+H_{3} \\
& +H_{4}+H_{5}+\ldots
\end{aligned}
$$

where

$$
H_{0}=H\left(\xi, \eta, p_{\xi}, p_{\eta}\right)=\text { constant } H_{1}=0 \text {. }
$$

Now, the Hamiltonian given by equation (3.4) is expanded using equation (3.5). The terms independent of $\mathrm{p}_{\mathrm{i}}, \mathrm{q}_{\mathrm{i}}$ and those having order more than one are neglected. Thus, the Hamiltonian is represented as follows:

$$
\begin{aligned}
& H=\frac{p_{1}^{2}+p_{2}^{2}}{2}+\left(p_{1} q_{2}-p_{2} q_{1}\right)+ \\
& \frac{\left(q_{1}^{2}+q_{2}^{2}\right) e \cos f}{2(1+e \cos f)}-\frac{1}{1+e \cos f} \times \\
& \left\{(1-\mu)\left(1-\beta_{1}\right)\left(\bar{r}_{1}\right)^{-1}+\mu\left(1-\beta_{2}\right)\left(\bar{r}_{2}\right)^{-1}\right\}
\end{aligned}
$$

After further mathematical manipulations and using Taylor's theorem the Hamiltonian is given as follows:

$$
\begin{aligned}
& H=\frac{p_{1}^{2}+p_{2}^{2}}{2}+\left(p_{1} q_{2}-p_{2} q_{1}\right) \\
& +\frac{\left(q_{1}^{2}+q_{2}^{2}\right) e \cos f}{2(1+e \cos f)}- \\
& {\left[\left\{q_{1}\left(\frac{-1}{2}-\frac{\beta_{1}}{6}-\frac{\beta_{2}}{3}\right)+q_{2}\left(\frac{-\sqrt{3}}{2}-\frac{7 \beta_{1}}{6 \sqrt{3}}+\frac{\beta_{2}}{3 \sqrt{3}}\right)\right\}+\right.} \\
& \frac{1}{2}\left\{q_{1}^{2}\left(\frac{-1}{4}-\frac{3 \beta_{1}}{4}+\beta_{2}\right)+2 q_{1} q_{2}\left(\frac{3 \sqrt{3}}{4}+\frac{7 \beta_{1}}{4 \sqrt{3}}+\frac{\beta_{2}}{\sqrt{3}}\right)\right. \\
& \left.+q_{2}{ }^{2}\left(\frac{5}{4}+\frac{7 \beta_{1}}{4}-\beta_{2}\right)\right\}+\frac{1}{6}\left\{q_{1}^{3}\left(\frac{21}{8}+\frac{31 \beta_{1}}{8}-\frac{3 \beta_{2}}{4}\right)+\right. \\
& +3 q_{1}^{2} q_{2}\left(\frac{-3 \sqrt{3}}{8}+\frac{17 \beta_{1}}{8 \sqrt{3}}-\frac{58 \sqrt{3} \beta_{2}}{8 \sqrt{3}}\right) \\
& +3 q_{1} q_{2}^{2}\left(\frac{-33}{8}-\frac{43 \beta_{1}}{8}-\frac{\beta_{2}}{4}\right) \\
& \left.+q_{2}^{3}\left(-\frac{9 \sqrt{3}}{8}-\frac{23 \sqrt{3} \beta_{1}}{8}+\frac{11 \sqrt{3} \beta_{2}}{4}\right)\right\} \\
& +\frac{1}{24}\left\{q_{1}^{4}\left(\frac{-111}{16}-\frac{85 \beta_{1}}{16}+\frac{25 \beta_{2}}{2}\right)\right. \\
& +4 q_{1}^{3} q_{2}\left(\frac{-75}{16}-\frac{535 \sqrt{3} \beta_{1}}{48}+\frac{20 \sqrt{3} \beta_{2}}{3}\right) \\
& +6 q_{1}^{2} q_{2}^{2}\left(\frac{123}{16}+\frac{65 \beta_{1}}{16}+\frac{35 \beta_{2}}{2}\right) \\
& +4 q_{1} q_{2}^{3}\left(\frac{135 \sqrt{3}}{16}-\frac{265 \sqrt{3} \beta_{1}}{16}-5 \sqrt{3} \beta_{2}\right) \\
& \left.\left.+q_{2}^{4}\left(\frac{9}{16}+\frac{195 \beta_{1}}{16}-\frac{45 \beta_{2}}{2}\right)\right\}\right] \\
& -\frac{\mu\left(1-\beta_{2}\right)}{1+e \cos f}\left(1+\frac{\beta_{2}}{3}\right)-\frac{\mu\left(1-\beta_{2}\right)}{1+e \cos f} \times
\end{aligned}
$$$$
\left[\left\{q_{1}\left(\frac{1}{2}+\frac{\beta_{1}}{3}+\frac{\beta_{2}}{6}\right)+q_{2}\left(\frac{-\sqrt{3}}{2}+\frac{\beta_{1}}{3 \sqrt{3}}-\frac{7 \beta_{2}}{6 \sqrt{3}}\right)\right\}+\right.
$$$$
\frac{1}{2}\left\{q_{1}^{2}\left(\frac{-1}{4}+\beta_{1}-\frac{3 \beta_{2}}{4}\right)+\right.
$$$$
2 q_{1} q_{2}\left(\frac{-3 \sqrt{3}}{4}-\frac{\beta_{1}}{\sqrt{3}}-\frac{7 \beta_{2}}{4 \sqrt{3}}\right)
$$$$
\left.+q_{2}{ }^{2}\left(\frac{5}{4}-\beta_{1}+\frac{7 \beta_{2}}{4}\right)\right\}
$$$$
+\frac{1}{6}\left\{q_{1}^{3}\left(\frac{-21}{8}+\frac{3 \beta_{1}}{4}-\frac{31 \beta_{2}}{8}\right)\right.
$$$$
+3 q_{1}^{2} q_{2}\left(\frac{-3 \sqrt{3}}{8}-\frac{58 \beta_{1}}{8 \sqrt{3}}+\frac{17 \beta_{2}}{8 \sqrt{3}}\right)+
$$$$
3 q_{1} q_{2}^{2}\left(\frac{33}{8}+\frac{\beta_{1}}{4}+\frac{43 \beta_{2}}{8}\right)
$$$$
\left.+q_{2}^{3}\left(\frac{-9 \sqrt{3}}{8}+\frac{11 \sqrt{3} \beta_{1}}{4}-\frac{23 \sqrt{3} \beta_{2}}{8}\right)\right\}
$$$$
+\frac{1}{24}\left\{q_{1}^{4}\left(\frac{-111}{16}-\frac{25 \beta_{1}}{2}-\frac{85 \beta_{2}}{16}\right)+\right.
$$$$
+4 q_{1}^{3} q_{2}\left(\frac{75 \sqrt{3}}{16}-\frac{20 \sqrt{3} \beta_{1}}{3}+\frac{535 \sqrt{3} \beta_{2}}{48}\right)
$$$$
+6 q_{1}^{2} q_{2}^{2}\left(\frac{123}{16}+\frac{35 \beta_{1}}{2}+\frac{65 \beta_{2}}{16}\right)+
$$$$
+4 q_{1} q_{2}^{3}\left(\frac{-135 \sqrt{3}}{16}+5 \sqrt{3} \beta_{1}-\frac{265 \sqrt{3}}{16} \beta_{2}\right)
$$$$
\left.\left.+q_{2}^{4}\left(\frac{9}{16}-\frac{45 \beta_{1}}{2}+\frac{195 \beta_{2}}{16}\right)\right\}\right]
$$

Equating the coefficient of $2^{\text {nd }}, 3^{\text {rd }}, 4^{\text {th }}$ order we get:

$$
\begin{aligned}
& H_{2}=\frac{p_{1}^{2}+p_{2}^{2}}{2}+\left(p_{1} q_{2}-p_{2} q_{1}\right)+ \\
& \frac{\left(q_{1}^{2}+q_{2}^{2}\right) e \cos f}{2(1+e \cos f)}+\frac{1}{(1+e \cos f)} \times \\
& \quad\left[\left(\frac{1}{8}+A\right) q_{1}^{2}-q_{1} q_{2}(K-B)-q_{2}^{2}\left(\frac{5}{8}+A\right)\right]
\end{aligned}
$$

where

$$
\begin{aligned}
& A=\frac{\beta_{1}}{4}-\frac{\beta_{2}}{2}-\frac{3 \mu \beta_{1}}{4}+\frac{3 \mu \beta_{2}}{4} \\
& B=\frac{3 \sqrt{3}}{4}\left(\frac{2 \beta_{1}}{9}-\frac{4 \beta_{2}}{9}+\frac{2 \mu \beta_{1}}{9}+\frac{2 \mu \beta_{2}}{9}\right) \\
& K=\frac{3 \sqrt{3}(1-2 \mu)}{4}
\end{aligned}
$$

For sake of convenience, let us substitute:

$$
H_{20}=\frac{1}{8}+A ; H_{11}=K-B ; H_{02}=\frac{5}{8}+A
$$


The equation (3.12) reduces to the form:

$$
\begin{aligned}
& H_{2}=\frac{p_{1}^{2}+p_{2}^{2}}{2}+\left(p_{1} q_{2}-p_{2} q_{1}\right)+\frac{e \cos f}{2(1+e \cos f)} \\
& \times\left(q_{1}^{2}+q_{2}^{2}\right)-\frac{1}{2(1+e \cos f)} \times \\
& \left(H_{20} q_{1}^{2}+H_{11} q_{1} q_{2}+H_{02} q_{2}^{2}\right)
\end{aligned}
$$

where,

$$
\begin{aligned}
& H_{20}=\frac{1}{8}+\frac{\beta_{1}}{4}-\frac{\beta_{2}}{2}-\frac{3 \mu \beta_{1}}{4}+\frac{3 \mu \beta_{2}}{4} \\
& H_{11}=\frac{3 \sqrt{3}}{4}-\frac{\beta_{1}}{2 \sqrt{3}}-\frac{\sqrt{3} \beta_{2}}{3}-\frac{3 \sqrt{3} \mu}{2} \\
& -\frac{\sqrt{3} \mu \beta_{1}}{6}-\frac{\sqrt{3} \mu \beta_{2}}{6} \\
& H_{02}=\frac{5}{8}+\frac{\beta_{1}}{4}-\frac{\beta_{2}}{2}-\frac{3 \mu \beta_{1}}{4}+\frac{3 \mu \beta_{2}}{4}
\end{aligned}
$$

$$
\begin{aligned}
& H_{3}=\frac{-1}{6(1+e \cos f)} \times \\
& {\left[q_{1}^{3}\left(\frac{21}{8}+\frac{5 \beta_{1}}{4}-\frac{3}{4} \beta_{2}-\frac{21 \mu}{4}-\frac{\mu \beta_{1}}{2}-\frac{\mu \beta_{2}}{2}\right)\right.} \\
& +3 q_{1}^{2} q_{2}\left(-\frac{3 \sqrt{3}}{8}-\frac{26 \sqrt{3} \beta_{1}}{8}-\frac{29 \sqrt{3} \beta_{2}}{12}\right. \\
& \left.\quad-\frac{138 \sqrt{3} \mu \beta_{1}}{24}+\frac{84 \sqrt{3} \mu \beta_{2}}{24}\right) \\
& +3 q_{1} q_{2}^{2}\left(-\frac{33}{8}-\frac{5 \beta_{1}}{4}-\frac{\beta_{2}}{4}+\frac{33 \mu}{4}\right. \\
& \left.+\frac{3 \mu \beta_{1}}{2}+\frac{3 \mu \beta_{2}}{2}\right)+ \\
& q_{2}^{3}\left(\frac{-9 \sqrt{3}}{8}-\frac{14 \sqrt{3} \beta_{1}}{8}+\frac{11 \sqrt{3}}{12} \beta_{2}\right. \\
& \left.\left.\quad+\frac{25 \sqrt{3} \mu \beta_{1}}{4}-\frac{25 \sqrt{3} \mu \beta_{2}}{4}\right)\right]
\end{aligned}
$$

$$
\begin{aligned}
& H_{4}=\frac{-1}{24(1+e \cos f)} \times \\
& {\left[q_{1}^{4}\left(\frac{-111}{16}+\frac{13 \beta_{1}}{8}+\frac{25 \beta_{2}}{2}-\frac{113 \mu \beta_{1}}{8}-\frac{87 \mu \beta_{2}}{8}\right)\right.} \\
& +4 q_{1}{ }^{3} q_{2}\left(-\frac{75 \sqrt{3}}{16}-\frac{310 \sqrt{3} \beta_{1}}{48}+\frac{20 \sqrt{3} \beta_{2}}{3}+\right. \\
& \left.\quad \frac{75 \sqrt{3} \mu}{8}-\frac{10 \sqrt{3} \mu \beta_{1}}{48}-\frac{10 \sqrt{3} \mu \beta_{2}}{48}\right) \\
& +6 q_{1}{ }^{2} q_{2}{ }^{2}\left(\frac{123}{16}-\frac{29 \beta_{1}}{16}+\frac{35 \beta_{2}}{2}\right. \\
& \left.+\frac{169 \mu \beta_{1}}{8}-\frac{169 \mu \beta_{2}}{8}\right)+ \\
& 4 q_{1} q_{2}{ }^{3}\left(-\frac{135 \sqrt{3}}{16}+\frac{130 \sqrt{3} \beta_{1}}{16}-5 \sqrt{3} \beta_{2}\right. \\
& \left.\quad-\frac{135 \sqrt{3} \mu}{8}-\frac{50 \sqrt{3} \mu \beta_{1}}{16}-\frac{50 \sqrt{3} \mu \beta_{2}}{16}\right) \\
& +q_{2}^{4}\left(\frac{9}{16}+\frac{186 \beta_{1}}{16}-\frac{45}{2} \beta_{2}\right. \\
& \left.\left.\quad-\frac{273 \mu \beta_{1}}{8}+\frac{273 \mu \beta_{2}}{8}\right)\right]
\end{aligned}
$$

\section{Existence of resonance in circular case.}

The existence of resonance for $\omega_{1}=\omega_{2}, \omega_{1}=2 \omega_{2}, \omega_{1}=3 \omega_{2}$ has been analyzed in the model of elliptical restricted three body problem under the radiating primaries for the particular case, $\mathrm{e}=0$. The Hamiltonian for the circular problem up to the second order term is obtained, which is expressed as follows:

$$
\begin{aligned}
& H_{2}=\frac{p_{1}^{2}+p_{2}^{2}}{2}+\left(p_{1} q_{2}-p_{2} q_{1}\right)+ \\
& \left\{H_{20} q_{1}^{2}-q_{1} q_{2} H_{11}-H_{02} q_{2}^{2}\right\}
\end{aligned}
$$

where $H_{20}, H_{11}$ and $H_{02}$ are defined by the equations (3.16).

If $\mathrm{H}_{2}$ is of positive definite form, then the equilibrium points are stable for all orders as given by Liapunov's (1956). Otherwise stability can be investigated by Arnold's theorem [1963(a, b)] .In order to apply Arnold's theorem, H2 is normalized using linear transformation of variables as given by Markeev (1978). Restricting to $\mathrm{H}_{2}$ and using the canonical transformation the characteristics equation takes the following form:

$$
\left|\begin{array}{cc}
\lambda^{2}-\left(1-2 H_{20}\right) & -2 \lambda-H_{11} \\
2 \lambda-H_{11} & \lambda^{2}-\left(1+2 H_{02}\right)
\end{array}\right|=0
$$

After further calculations the characteristics equation reduces to the form:

$$
\lambda^{4}+\lambda^{2}+\left\{\frac{27 \mu(1-\mu)}{4}\right\}\left\{1+\frac{2 \beta_{1}}{9}+\frac{2 \beta_{2}}{9}\right\}=0
$$

If $\omega_{1}$ and $\omega_{2}$ be the frequencies then we put $\lambda^{2}=-\omega^{2}$ and the equation satisfied by the frequencies is given as:

$$
\omega^{4}-\omega^{2}+\left\{\frac{27 \mu(1-\mu)}{4}\right\}\left\{1+\frac{2 \beta_{1}}{9}+\frac{2 \beta_{2}}{9}\right\}=0
$$


If $\omega_{1}, \omega_{2}$ be the frequencies corresponding to the equation (4.4) then $\omega_{1}^{2}=-\lambda_{1,2}^{2}$ and $\omega_{2}^{2}=-\lambda_{3,4}^{2}$

We discussed the following cases:

Case1. Assuming $\omega_{1}=\omega_{2}$ i.e. $\lambda_{1,2}^{2}=\lambda_{3,4}^{2}$

I.e. roots of the characteristics equation are equal. Therefore, discriminant is equal to zero.i.e.

$$
1-27 \mu(1-\mu)\left(1+2 \beta_{1} / 9+2 \beta_{2} / 9\right)=0
$$

i.e.

$$
27\left(1+\frac{2 \beta_{1}}{9}+\frac{2 \beta_{2}}{9}\right) \mu^{2}-27\left(1+\frac{2 \beta_{1}}{9}+\frac{2 \beta_{2}}{9}\right) \mu+1=0
$$

Since $\mu \leq \frac{1}{2}$, the positive sign is inadmissible. Hence, the region of stability in the first approximation can be written as:

$0<\mu<\frac{\left\{9-\sqrt{69}\left(1+\frac{4 \beta_{1}}{207}+\frac{4 \beta_{2}}{207}\right)\right\}}{18}$

Thus, the value of $\mu$ responsible for stable equilibrium points is given by:

$$
\mu_{01}=0.03852088-0.008917 \beta_{1}-0.008917 \beta_{2}
$$

When $\beta_{1}, \beta_{2}$ vanishes, $\mu_{\text {critical }}=0.03852088$,

Case2. Assuming $\omega_{1}=2 \omega_{2}$ i.e. $\lambda_{1,2}^{2}=4 \lambda_{3,4}^{2}$ i.e.

$$
675\left(1+\frac{2 \beta_{1}}{9}+\frac{2 \beta_{2}}{9}\right) \mu^{2}-675\left(1+\frac{2 \beta_{1}}{9}+\frac{2 \beta_{2}}{9}\right) \mu+16=0
$$

Solving for $\mu$, the resonance value is obtained as:

$$
\mu_{02}=0.02429396-0.00553648 \beta_{1}-0.00553648 \beta_{2}
$$

Case3.If $\omega_{1}=3 \omega_{2}$ i.e. $\lambda_{1,2}^{2}=9 \lambda_{3,4}^{2}$

i.e.

$$
675\left(1+\frac{2 \beta_{1}}{9}+\frac{2 \beta_{2}}{9}\right) \mu^{2}-675\left(1+\frac{2 \beta_{1}}{9}+\frac{2 \beta_{2}}{9}\right) \mu+9=0
$$

$\mu_{03}=0.0135167-0.0029629 \beta_{1}-0.0029629 \beta_{2}$

The critical value $\mu_{c}$ usually corresponds to the boundary of the region of stability of the linear system and hence the case is not being considered in our further investigation. Taking into consideration of the range of stability, it is found that the range which we defined for, $\mu_{c}<0.0385208$ the condition $\omega_{1}=\omega_{2}$, will not be satisfied .The condition of the range of stability is satisfied for $\omega_{1}=\omega_{2}$, when $\mu_{c}>0.0385208$

From equation (4.11) and (4.13) it is clear that resonance is possible for $\omega_{1}=2 \omega_{2}, \omega_{1}=3 \omega_{2}$

Figs.1-5 are representing the correlation between $\mu$ and $\omega_{1}, \omega_{2}$ for $\mathrm{e}=0$

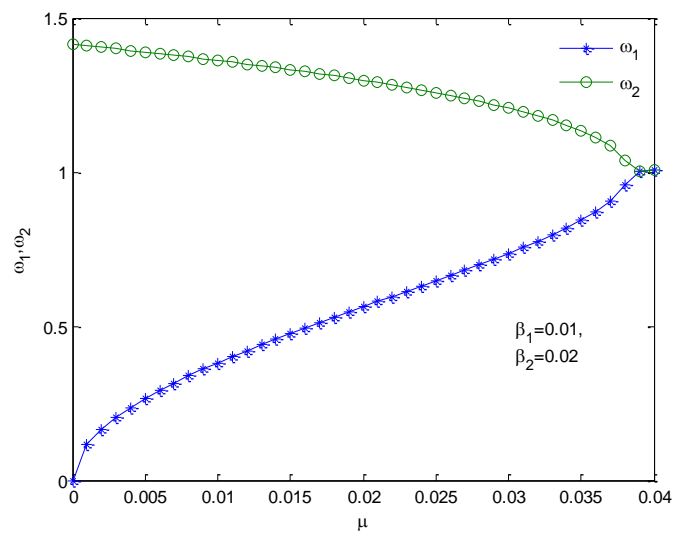

Fig. 1: Correlation between $\mu$ and $\omega_{1}, \omega_{2}$ for $e=0$ when $\beta_{1}=.01, \beta_{2}=0.02$.

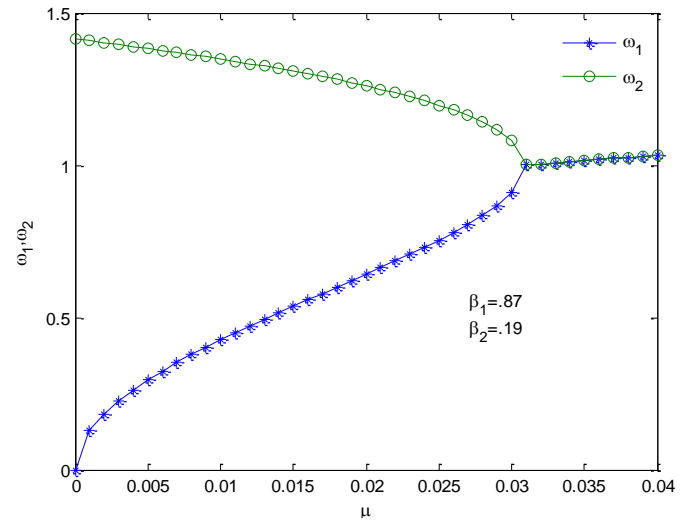

Fig. 2: Correlation between $\mu$ and $\omega_{1}, \omega_{2}$ for $\mathrm{e}=0$ when $\beta_{1}=0.87, \beta_{2}=0.19$

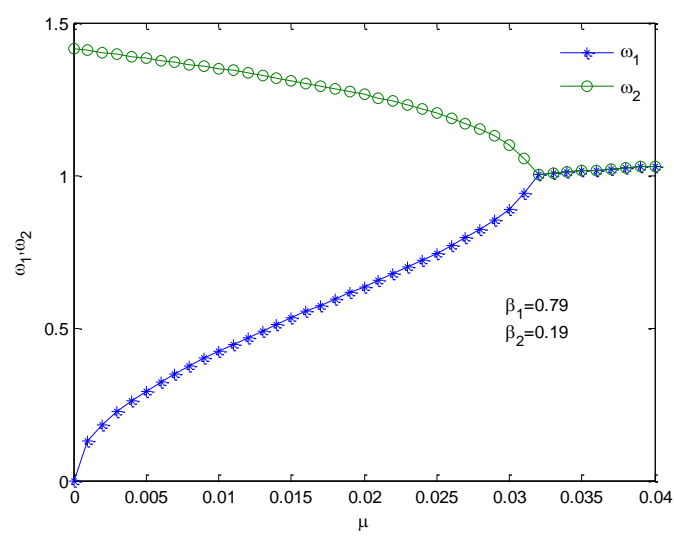

Fig. 3: Correlation between $\mu$ and $\omega_{1}, \omega_{2}$.for $e=0$ when $\beta_{1}=0.79, \beta_{2}=0.29$.

Solving for $\mu$, the resonance value is obtained as: 


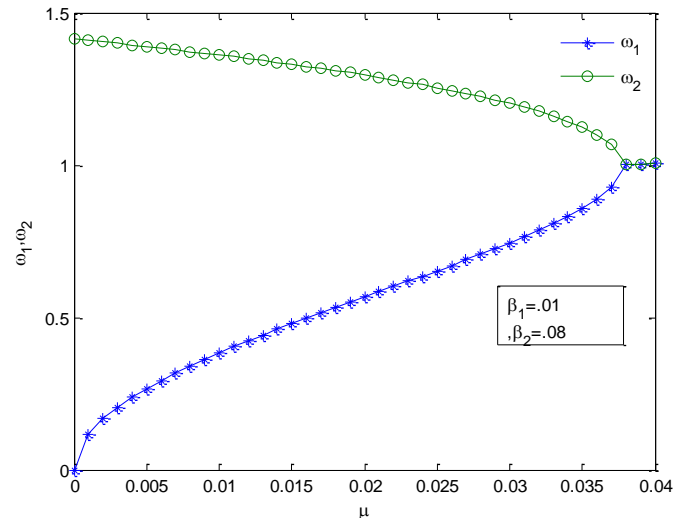

Fig. 4: Correlation between $\mu$ and $\omega_{1}, \omega_{2}$ for $e=0$ when $\beta_{1}=0.01, \beta_{2}=0.08$

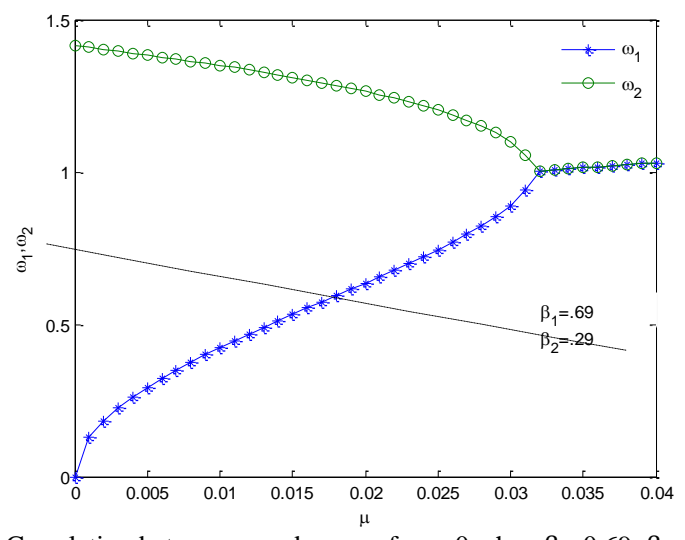

Fig. 5: Correlation between $\mu$ and $\omega_{1}, \omega_{2}$ for $e=0$ when $\beta_{1}=0.69, \beta_{2}=0.29$

The correlation between $\beta_{2}$ and $\mu$ for circular case for various values of radiation pressure is shown in fig. 6-13.

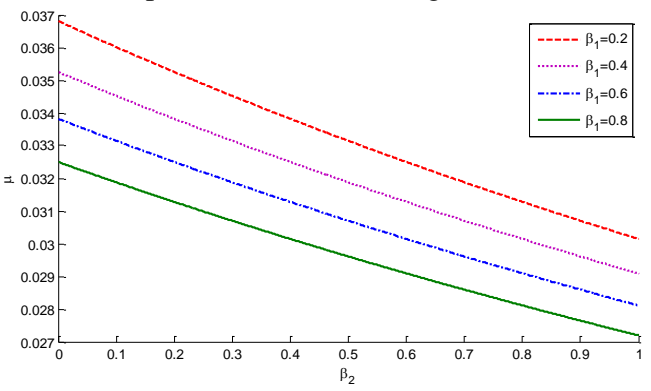

Fig. 6: Correlation between $\beta_{2}$ and $\mu$ when $\omega_{1}=\omega_{2}$ for $e=0$

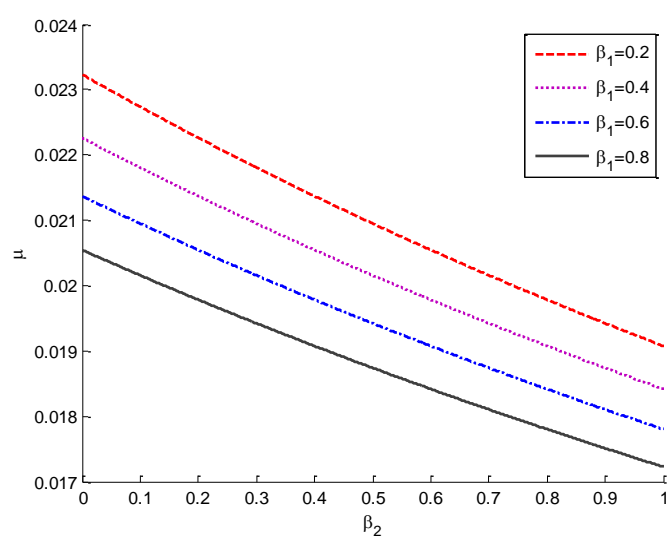

Fig. 7: Correlation between $\beta_{2}$ and $\mu$ when $\omega_{1}=2 \omega_{2}$ for $e=0$

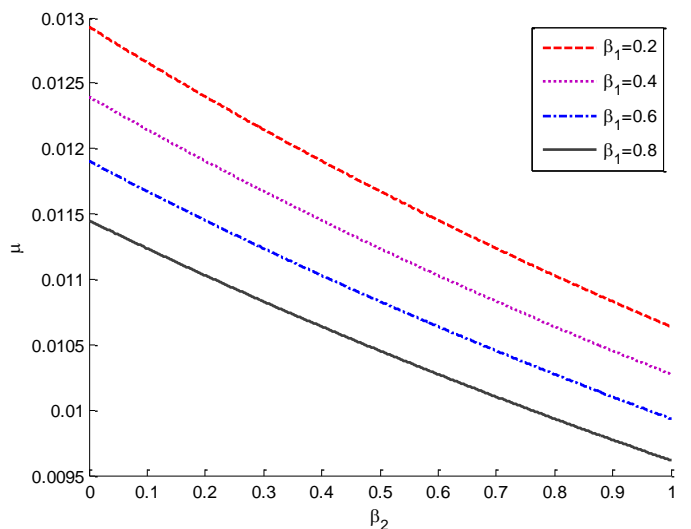

Fig. 8: Correlation between $\beta_{2}$ and $\mu$ when $\omega_{1}=3 \omega_{2}$ for $e=0$

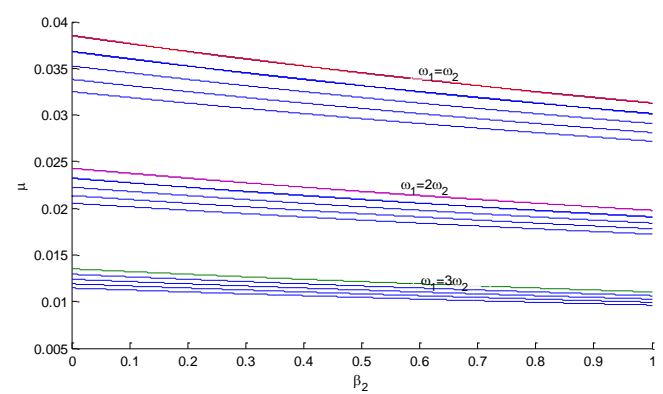

Fig. 9: Combined Graph of Fig .6, 7, 8

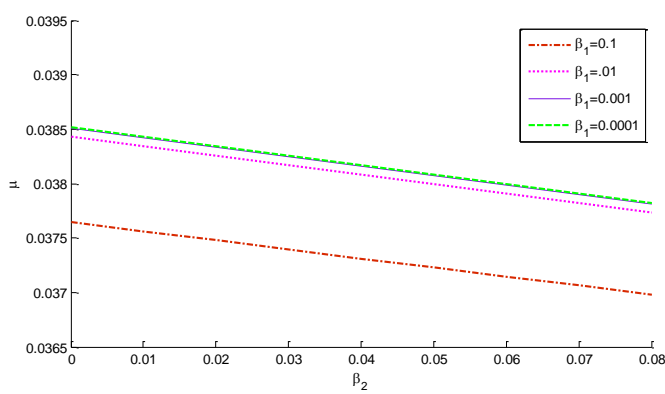

Fig. 10: Correlation between $\beta_{2}$ and $\mu$ when $\omega_{1}=\omega_{2}$ for $e=0$

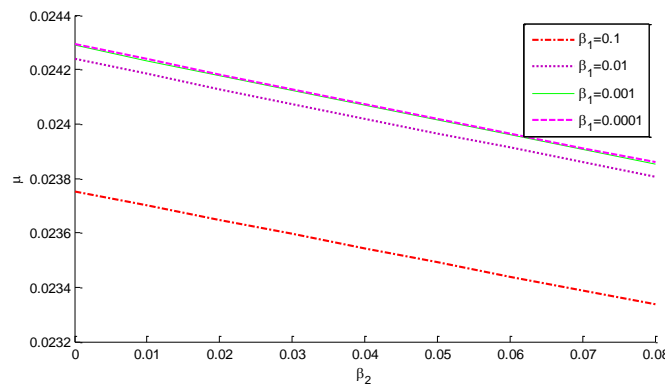

Fig. 11: Correlation between $\beta_{2}$ and $\mu$ when $\omega_{1}=2 \omega_{2}$ for $e=0$

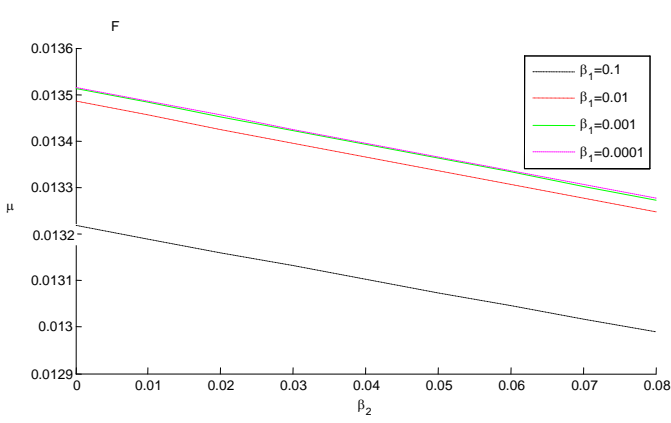

Fig. 12: Correlation between $\beta_{2}$ and $\mu$ when $\omega_{1}=3 \omega_{2}$ for $e=0$ 


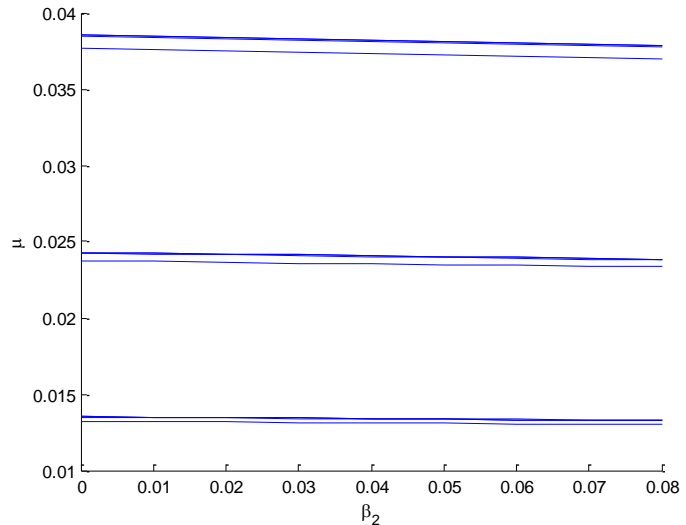

Fig. 13: Combined Graph of Fig 10, 11, 12

\section{Existence of resonance in elliptical case.}

Considering equation (3.5) and expanding the Hamiltonian function $\mathrm{H}_{2}$ in powers of 'e' up to the first approximation we get:

$$
\begin{aligned}
& H_{2}=\frac{p_{1}{ }^{2}+p_{2}{ }^{2}}{2}+\left(P_{1} q_{2}-P_{2} q_{1}\right)+ \\
& {\left[\left(\frac{1}{8}+A\right) q_{1}{ }^{2}-(k-B) q_{1} q_{2}-\left(\frac{5}{8}+A\right) q_{2}{ }^{2}\right]} \\
& +(e \cos f) \times\left[\left(\frac{3}{8}-A\right) q_{1}{ }^{2}+(k-B) q_{1} q_{2}+\left(\frac{9}{8}+A\right) q_{2}{ }^{2}\right]
\end{aligned}
$$

The characteristics equation in this case is given by:

$$
\begin{aligned}
& \lambda^{4}+\left(1+\frac{e^{2}}{2}\right) \lambda^{2}+\left\{\frac{27 \mu(1-\mu)}{4}\right\} \times \\
& \left\{1+\frac{2 \beta_{1}}{9}+\frac{2 \beta_{2}}{9}\right\}\left(1+e^{2}\right)=0
\end{aligned}
$$

Proceeding in the same manner as in section 4, the roots of the characteristics equation are represented as:

$\omega_{1}^{2}=-\lambda_{1,2}^{2}$

$\omega_{1}^{2}=\frac{1}{2}\left[\left(1+\frac{e^{2}}{2}\right)-\left\{\begin{array}{l}\left(1+\frac{e^{2}}{2}\right)^{2}-27 \mu(1-\mu) \times\left(1+e^{2}\right) \\ \times\left(1+\frac{2 \beta_{1}}{9}+\frac{2 \beta_{2}}{9}\right)\end{array}\right\}^{1 / 2}\right]$

$\omega_{2}^{2}=-\lambda_{3,4}^{2}$

$\omega_{2}^{2}=\frac{1}{2}\left[\left(1+\frac{e^{2}}{2}\right)+\left\{\begin{array}{l}\left(1+\frac{e^{2}}{2}\right)^{2}-27 \mu(1-\mu) \times\left(1+e^{2}\right) \\ \times\left(1+\frac{2 \beta_{1}}{9}+\frac{2 \beta_{2}}{9}\right)\end{array}\right\}^{1 / 2}\right]$

Again considering the three cases as follows:

Case 1.Assuming, $\omega_{1}=\omega_{2}$ i.e. $\lambda_{1,2}^{2}=\lambda_{3,4}^{2}$

Then, solving for $\mu$, we get:
This value corresponds to the boundary of the stability region

Case2.

Let $\omega_{1}=2 \omega_{2}$ i.e. $\lambda_{1,2}^{2}=4 \lambda_{3,4}^{2}$

Hence, the value of $\mu$ is given as:

$$
\begin{aligned}
\mu_{02 e}= & 0.02429396-0.00553648 \beta_{1}- \\
& 0.00553648 \beta_{2}+e^{4}(0.0046714- \\
& \left.0.001038 \beta_{1}-0.001038 \beta_{2}\right)
\end{aligned}
$$

Case3.

Assuming $\omega_{1}=3 \omega_{2}$ i.e. $\lambda_{1,2}^{2}=9 \lambda_{3,4}^{2}$

Again, solving for $\mu$ we get:

$$
\begin{aligned}
& \mu_{03 e}=0.0135167-0.0029629 \beta_{1}-0.0029629 \beta_{2} \\
& +e^{4}\left(0.010277-0.002839 \beta_{1}-0.002839 \beta_{2}\right)
\end{aligned}
$$

Table-1and Table- 2 (see appendices) shows the values of $\mu$ corresponding to $\omega_{1}=\omega_{2}, \omega_{1}=2 \omega_{2}$ and $\omega_{1}=3 \omega_{2}$ satisfying the conditions $\beta_{1}+\beta_{2}>1 ; \beta_{1}, \beta_{2}<1$. and $\beta_{1}+\beta_{2}<1 ; \beta_{1}, \beta_{2}<1$ respectively.

It is clear from Table 1 and Table 2 that the corresponding values of $\mu$ are less than the critical value for every combination of the radiation pressures taken which states that the resonance condition exists for $\omega_{1}=2 \omega_{2}, \omega_{1}=3 \omega_{2}$ in both circular and elliptical cases.

Figs.14-18 show correlation between $\mu$ and $\omega_{1}, \omega_{2}$ for $\mathrm{e}=0.0489$.

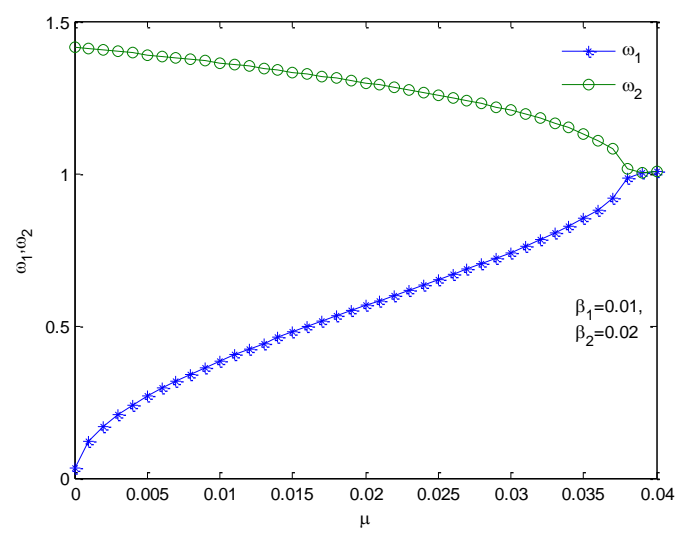

Fig. 14: Correlation between $\mu$ and $\omega_{1}, \omega_{2}$ for $e=0.0489$ when $\beta_{1}=.01$, $\beta_{2}=0.02$.

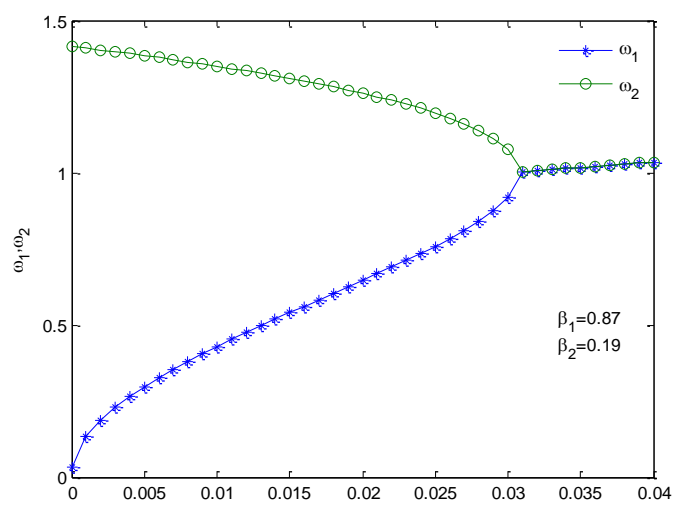

Fig. 15: Correlation between $\mu$ and $\stackrel{\mu}{\omega}, \omega_{2}$ for $e=0.0489$ when $\beta_{1}=0.87$, $\beta_{2}=0.19$.

$$
\begin{aligned}
\mu_{01 e}= & 0.03852088-0.008917 \beta_{1}- \\
& 0.008917 \beta_{2}+e^{4}(0.03009- \\
& \left.0.006687 \beta_{1}-0.006687 \beta_{2}\right)
\end{aligned}
$$




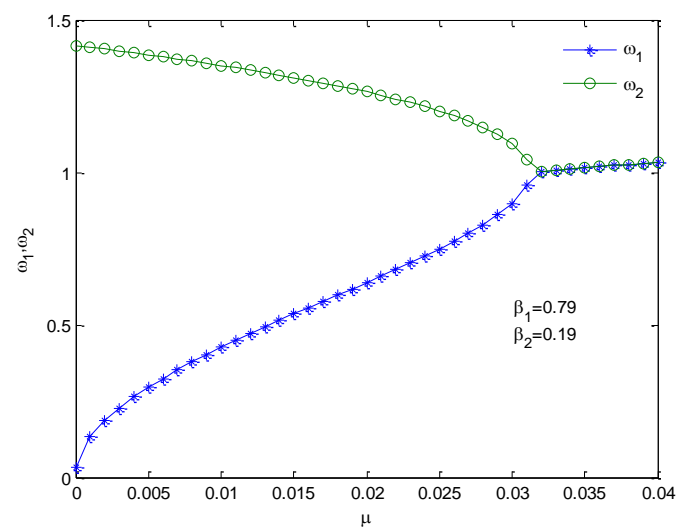

Fig. 16: Correlation between $\mu$ and $\omega_{1}, \omega_{2}$ for $e=0.0489$ when $\beta_{1}=0.79$, $\beta_{2}=0.19$.

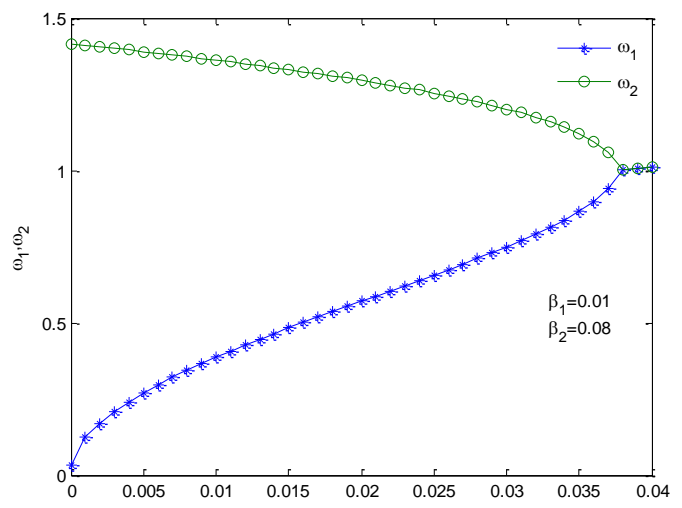

Fig. 17: Correlation between $\mu$ and $\omega_{1}, \omega_{2}$ for $e=0.0489$ when $\beta_{1}=0.01$, $\beta_{2}=0.08$.

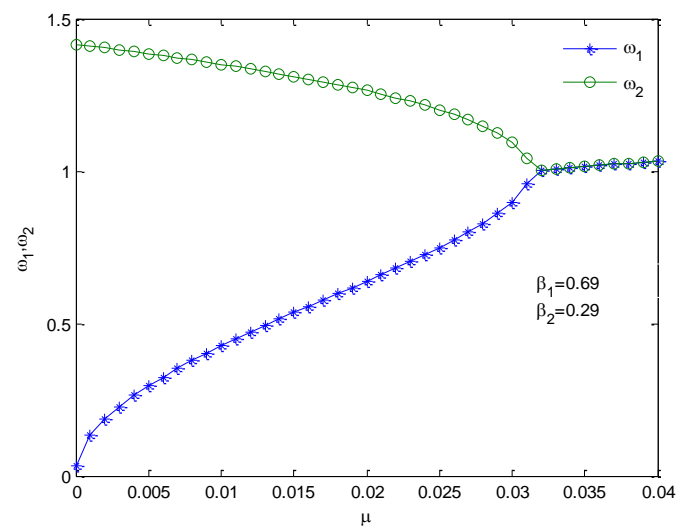

Fig. 18: Correlation between $\mu$ and $\omega_{1}, \omega_{2}$ for $e=0.0489$ when $\beta_{1}=0.69$, $\beta_{2}=0.29$.

Figs .19-22 are depicting correlation between e and $\mu$ for various values of radiation pressure.

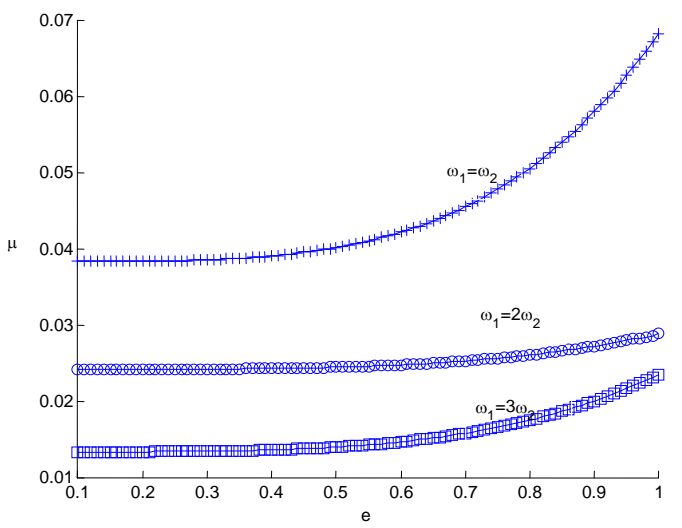

Fig. 19: Correlation between e and $\mu$ when $\beta_{1}=.01$ and $\beta_{2}=0.02$.

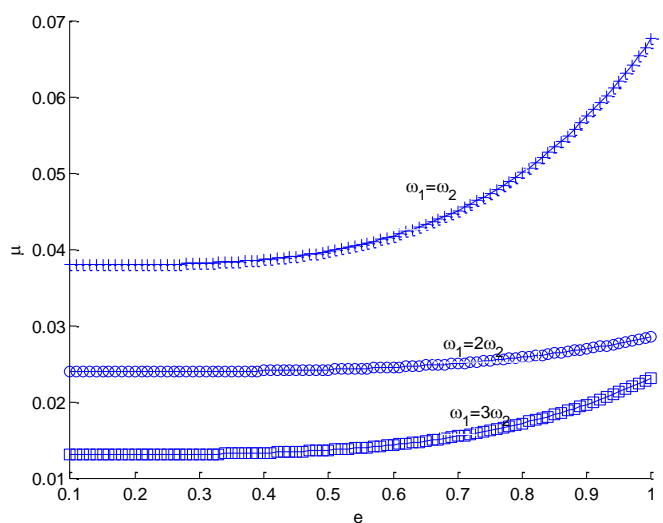

Fig .20: Correlation between e and $\mu$ when $\beta_{1}=.03$ and $\beta_{2}=0.04$.

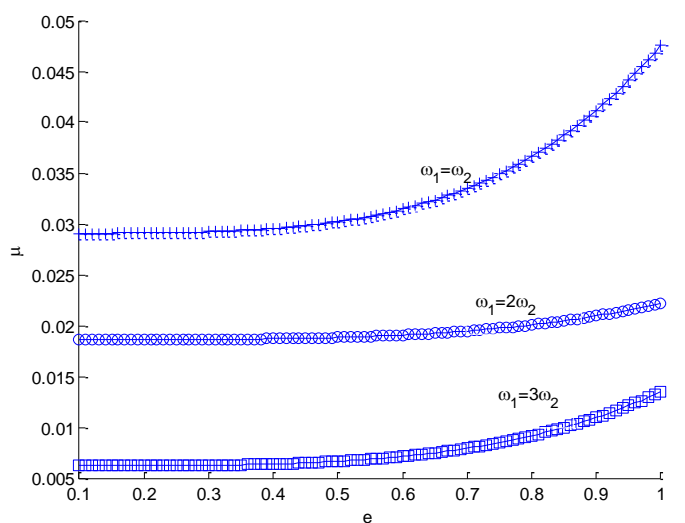

Fig .21: Correlation between e and $\mu$ when $\beta_{1}=0.87$ and $\beta_{2}=0.19$.

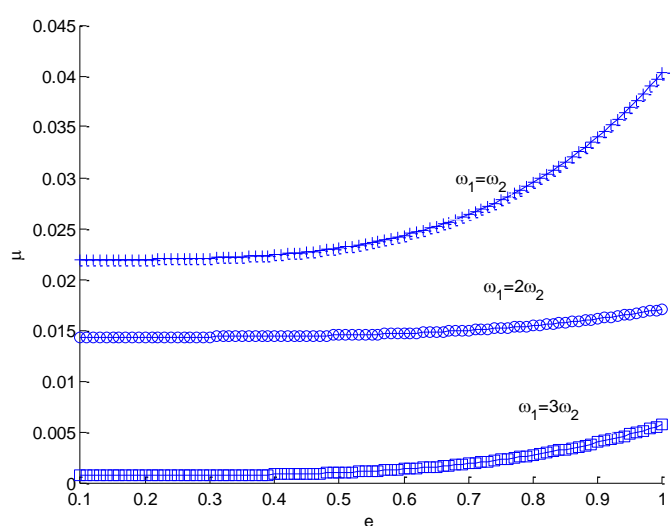

Fig .22: Correlation between e and $\mu$ when $\beta_{1}=0.87$ and $\beta_{2}=0.99$.

Figs 23-25 are representing correlation between $\beta_{2}$ and $\mu$ for $\mathrm{e}=0.0489$ which is shown below.

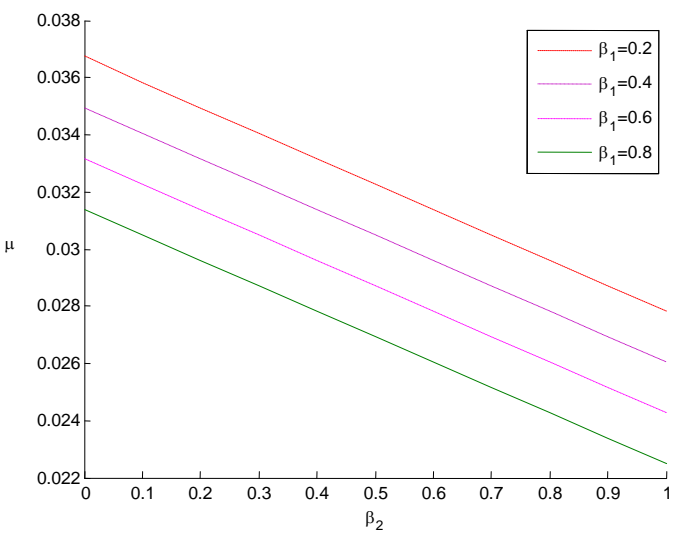

Fig. 23: Correlation between $\beta_{2}$ and $\mu$ when $\omega_{1}=\omega_{2}$ for $\mathrm{e}=0.0489$. 


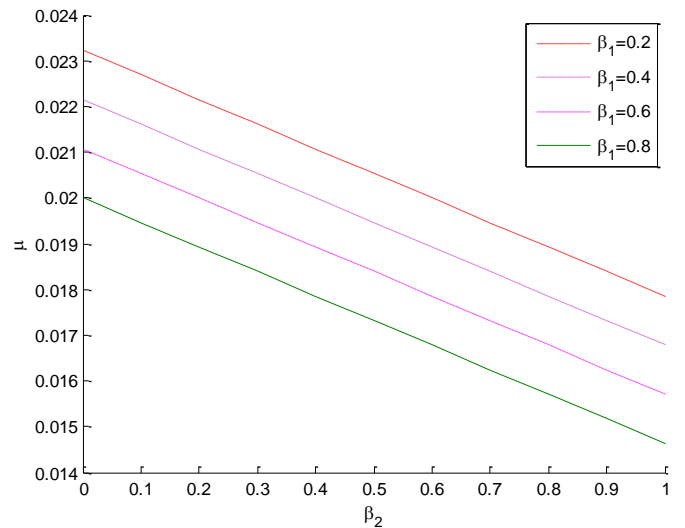

Fig. 24: Correlation between $\beta_{2}$ and $\mu$ when $\omega_{1}=2 \omega_{2}$ for $e=0.0489$.

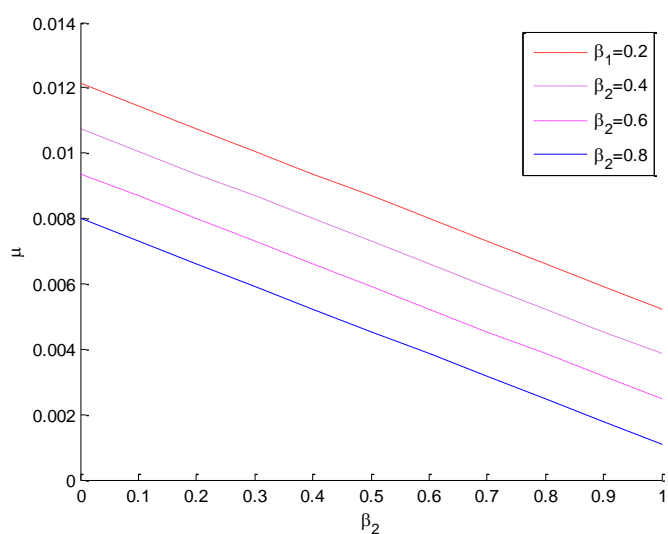

Fig. 25: Correlation between $\beta_{2}$ and $\mu$ when $\omega_{1}=3 \omega_{2}$ for $e=0.0489$.

\section{Normalization and higher order stability of the libration points}

In order to investigate the stability of the libration point, the Hamiltonian $\mathrm{H}$ is normalized by Birkhoff's method to the following form and then KAM theorem is applied.

$$
\begin{aligned}
H= & \omega_{1} r_{1}-\omega_{2} r_{2}+c_{20} r_{1}^{2}+c_{11} r_{1} r_{2}+c_{02} r_{2}^{2}+ \\
& o\left(r_{1}+r_{2}\right)^{5 / 2}
\end{aligned}
$$

In this section KAM theory is applied which can be stated as follows:

If the Hamiltonian of the perturbed motion is such that:

1) The characteristics equation of the system with $\mathrm{H}$ has purely imaginary roots,

2) $n_{1} \omega_{1}+n_{2} \omega_{2} \neq 0$, where $n_{1}, n_{2}$ are integers satisfying , $0<\left|n_{1}\right|+\left|n_{2}\right| \leq 4$,

3) $D=c_{20} \omega_{2}^{2}+c_{11} \omega_{1} \omega_{2}+c_{02} \omega_{1}^{2} \neq 0$,

Then, the equilibrium points are stable.

In order to analyse further we applied KAM theorem, for which linear canonical transformation of variations is used as given by:

$\left(q_{1}, q_{2}, p_{1}, p_{2}\right)=\left(q_{1}^{\prime}, q_{2}{ }_{2}, p^{\prime}{ }_{1}, p^{\prime}{ }_{2}\right) N$

Where,

$$
N=\left[\begin{array}{cccc}
a_{1} & a_{1} c_{1} & -a_{1} c_{1} & a_{1}\left(1-\omega_{1}^{2} b_{1}\right) \\
a_{2} & a_{2} c_{2} & -a_{2} c_{2} & a_{2}\left(1-\omega_{2}^{2} b_{2}\right) \\
0 & a_{1} b_{1} & a_{1}\left(1-b_{1}\right) & a_{1} c_{1} \\
0 & -a_{2} b_{2} & -a_{2}\left(1-b_{2}\right) & -a_{2} c_{2}
\end{array}\right]
$$

and

$a_{1}=\frac{1}{2}\left(\frac{2 l_{1}}{\omega_{1}^{2}-1 / 2}\right)^{1 / 2} ; a_{2}=\frac{1}{2}\left(\frac{2 l_{2}}{\omega_{2}^{2}-1 / 2}\right)^{1 / 2}$

$l_{1}=1+\omega_{1}^{2}+2 H_{02} ; \quad l_{2}=1+\omega_{2}^{2}+2 H_{02}$

$b_{1}=\frac{2}{l_{1}} ; \quad b_{2}=\frac{2}{l_{2}}$

$c_{1}=\frac{-H_{11}}{l_{1}} ; c_{2}=\frac{-H_{11}}{l_{2}}$

The transformation (6.2) reduces the Hamiltonian to the following form:

$$
\begin{aligned}
H= & \frac{1}{2}\left(p_{1}^{\prime 2}+\omega_{1}^{2} q_{1}^{\prime 2}\right)-\frac{1}{2}\left(p_{2}^{\prime 2}+\omega_{2}^{2} q_{2}^{\prime 2}\right)+ \\
& \sum_{\alpha+\delta=3}^{\infty} h_{\alpha_{1}, \alpha_{2} \delta_{1}, \delta_{2}} q_{1}^{\prime \alpha_{1}} q_{2}^{{ }^{\prime} \alpha_{2}} p_{1}^{\prime} \delta_{2} p_{2}{ }^{\prime \delta_{2}}
\end{aligned}
$$

where, $\alpha=\alpha_{1}+\alpha_{2} ; \delta=\delta_{1}+\delta_{2}$.

The Hamiltonian $\mathrm{H}_{3}$ can be expanded in the following form:

$$
\begin{aligned}
H_{3} & =H_{0003} p_{2}^{3}+H_{0030} p_{1}^{3}+H_{0300} q_{2}^{3} \\
& +H_{3000} q_{1}^{3}+H_{2100} q_{1}^{2} q_{2}+ \\
& H_{2010} q_{1}^{2} p_{1}+\ldots \ldots \ldots \ldots .+H_{1011} q_{1} p_{1} p_{2}
\end{aligned}
$$

Similarly $\mathrm{H}_{4}$ can be expanded as:

$$
H_{4}=H_{0004} p_{2}^{4}+H_{0040} p_{1}^{4}+H_{0400} q_{2}^{4}+
$$$$
H_{4000} q_{1}^{4}+\ldots \ldots \ldots \ldots . .+H_{1111} q_{1} q_{2} p_{1} p_{2}
$$

The coefficient of third and fourth order terms of $h_{\alpha_{1} \alpha_{2} \delta_{1} \delta_{2}}^{\prime}$ and $h_{\alpha_{1} \alpha_{2} \delta_{1} \delta_{2}}$ can be given as:

$h_{0030}=a_{1}^{3} b_{1}^{3} H_{0300}$;

$h_{3000}=a_{1}^{3}\left(H_{3000}+c_{1} H_{2100}+c_{1}^{2} H_{2100}\right.$

$\left.+c_{1}^{3} H_{0300}\right)$;

$h_{1020}=a_{1}^{3} b_{1}^{2}\left(H_{1200}+3 c_{1} H_{0300}\right) ;$

$h_{2010}=a_{1}^{3} b_{1}\left(H_{2100}+2 c_{1} H_{1200}+3 c_{1}^{2} H_{0300}\right)$;

$h_{0120}=a_{1}^{2} a_{2} b_{1}^{2}\left(H_{1200}+3 c_{2} H_{0300}\right) ;$

$h_{2001}=-a_{1}^{2} a_{2} b_{2}\left(H_{2100}+2 c_{1} H_{1200}\right.$

$$
\left.+3 c_{1}^{2} H_{0300}\right)
$$

$$
\begin{aligned}
h_{1011}= & -2 a_{1}^{2} a_{2} b_{1} b_{2}\left(H_{1200}+3 c_{1} H_{0300}\right) ; \\
h_{1110}= & 2 a_{1}^{2} a_{2} b_{1}\left[H_{2100}+\left(c_{1}+c_{2}\right) H_{1200}\right. \\
& \left.+3 c_{1} c_{2} H_{0300}\right] ; \\
h_{0021} & =-3 a_{1}^{2} b_{1}^{2} b_{2} H_{0300} ; \\
h_{2100} & =a_{1}^{2} a_{2}\left[3 H_{3000}+\left(2 c_{1}+c_{2}\right) H_{2100}+\right.
\end{aligned}
$$$$
\left.c_{1}\left(c_{1}+2 c_{2}\right) H_{1200}+3 c_{1}^{2} c_{2} H_{0300}\right]
$$ 


$$
\begin{aligned}
& h_{1002}=a_{1} b_{1}^{2} a_{2}^{2}\left(H_{1200}+3 c_{1} H_{0300}\right) \\
& h_{0210}=a_{1} a_{2}^{2} b_{1}\left(H_{2100}+2 c_{2} H_{1200}+3 c_{2}^{2} H_{0300}\right) \\
& h_{0012}=3 a_{1} a_{2}^{2} b_{2}^{2} b_{1} H_{0300} \\
& h_{1200}=a_{2}^{2} a_{1}\left[3 H_{3000}+\left(c_{1}+2 c_{2}\right) H_{2100}\right. \\
& \left.+c_{2}\left(2 c_{1}+c_{2}\right) H_{1200}+3 c_{1} c_{2}^{2} H_{0300}\right] \\
& h_{0111}=-2 a_{1}^{2} a_{2}^{2} b_{1} b_{2}\left(H_{1200}+3 c_{2} H_{0300}\right) \\
& h_{1101}=-2 a_{2}^{2} a_{1} b_{2}\left[H_{2100}+\left(c_{1}+c_{2}\right) H_{1200}\right. \\
& \left.+3 c_{1} c_{2} H_{0300}\right]
\end{aligned}
$$$$
h_{0102}=a_{2}^{3} b_{21}^{2}\left(H_{1200}+3 c_{2} H_{0300}\right) ; h_{0201}=-a_{2}^{3} b_{2}\left(H_{2100}+2 c_{2} H_{1200}+3 c_{2}^{2} H_{0300}\right) \text {; }
$$$$
h_{0102}=a_{2}^{3} b_{2}{ }^{2}\left(H_{1200}+3 c_{2} H_{0300}\right) ; h_{0003}=-a_{2}^{3} b_{2}{ }^{3} H_{0300} \text {; }
$$$$
h_{0300}=a_{2}^{3}\left(H_{3000}+c_{2} H_{2100}+c_{2}^{2} H_{1200}+h_{0040}=a_{1}^{4} b_{1}^{4} H_{0400}\right. \text {; }
$$$$
h_{4000}=a_{1}^{4}\left(H_{4000}+c_{1} H_{3100}+c_{1}^{2} H_{2200}+\right.
$$$$
\left.c_{1}^{3} H_{1300}+c_{1}^{4} H_{0400}\right) \text {; }
$$$$
h_{2020}=a_{1}^{4} b_{1}^{2}\left(H_{2200}+3 c_{1} H_{1300}+6 c_{1}^{2} H_{0400}\right) ; h_{0022}=6 a_{1}^{2} a_{2}^{2} b_{1}^{2} b_{2}^{2} H_{0400} ;
$$$$
h_{2200}=a_{1}^{2} a_{2}^{2}\left[6 H_{4000}+3\left(c_{1}+c_{2}\right) H_{3100}+\right.
$$$$
\left(c_{1}^{2}+4 c_{1} c_{2}+c_{2}^{2}\right) H_{2200}+3 c_{1} c_{2}\left(c_{1}+c_{2}\right) H_{1300}
$$$$
\left.+6 c_{1}^{2} c_{2}^{2} H_{0400}\right] \text {; }
$$

$$
\begin{aligned}
& h_{2002}=a_{1}^{2} a_{2}^{2} b_{2}^{2}\left(H_{2200}+3 c_{1} H_{1300}+6 c_{1}^{2} H_{0400}\right) ; \\
& h_{0220}=a_{1}^{2} a_{2}^{2} b_{1}^{2}\left(H_{2200}+3 c_{2} H_{1300}+6 c_{2}^{2} H_{0400}\right) ; \\
& h_{0004}=a_{2}^{4} b_{2}^{4} H_{0400} ; \\
& h_{0400}=a_{2}^{4}\left(H_{4000}+c_{2} H_{3100}+c_{2}^{2} H_{2200}\right. \\
& \left.+c_{2}^{3} H_{1300}+c_{2}^{4} H_{0400}\right) ;
\end{aligned}
$$$$
h_{0202}=a_{2}^{4} b_{2}{ }^{2}\left(H_{2200}+3 c_{2} H_{1300}+6 c_{2}^{2} H_{0400}\right) ; h_{0013}=-4 a_{1} a_{2}^{3} b_{1} b_{2}^{3} H_{0400}
$$$$
h_{1300}=a_{1} a_{2}^{3}\left[4 H_{4000}+\left(c_{1}+3 c_{2}\right) H_{3100}+\right.
$$$$
4 c_{1} c_{2}^{3} H_{0400}+2 c_{2}\left(c_{1}+c_{2}\right) H_{2200}
$$$$
\left.+c_{2}^{2}\left(3 c_{1}+c_{2}\right) H_{1300}\right] \text {; }
$$$$
h_{1102}=a_{1} a_{2}{ }^{3} b_{2}^{2}\left[2 H_{2200}+3\left(c_{1}+c_{2}\right) H_{1300}\right.
$$$$
\left.+12 c_{1} c_{2} H_{0400}\right] \text {; }
$$$$
h_{0211}=-2 a_{2}^{3} a_{1} b_{1} b_{2}\left[H_{2200}+3 c_{2} H_{1300}\right.
$$$$
+6 c_{2}{ }^{2} H_{0400} \text {; }
$$$$
h_{0112}=3 a_{1} a_{2}^{3} b_{1} b_{2}^{2}\left(H_{1300}+4 c_{1} H_{0400}\right)
$$

$h_{1003}=-a_{1} a_{2}^{3} b_{2}^{3}\left(H_{1300}+4 c_{1} H_{0400}\right)$;

$h_{1201}=-a_{1} a_{2}^{3} b_{2}\left[3 H_{3100}+2\left(c_{1}+2 c_{2}\right) H_{2200}\right.$

$\left.+3 c_{2}\left(2 c_{1}+c_{2}\right) H_{1300}+12 c_{1} c_{2}^{2} H_{0400}\right]$;

$h_{0310}=a_{1} a_{2}^{3} b_{1}\left(H_{3100}+2 c_{2} H_{2200}\right.$

$\left.+3 c_{2}^{2} H_{1300}+4 c_{2}^{2} H_{0400}\right)$;

Again the Hamiltonian is reduced to a more convenient form which is suitable for further investigation by using the following canonical transformation:

$q_{1}^{\prime}=\frac{1}{2} q_{1}^{\prime}+\frac{i}{\omega_{1}} p_{1}^{\prime \prime}$

$p_{1}^{\prime}=\frac{1}{2} i \omega_{1} q^{\prime \prime}+p{ }_{1}$

$q_{2}^{\prime}=-\frac{i}{2} q_{2}^{\prime \prime}+\frac{1}{\omega_{2}} p{ }_{2}$

$p_{2}^{\prime}=-\frac{1}{2} \omega_{2} q{ }_{2}+i p_{2}$

Thus, the Hamiltonian (6.5) may be written as:

$$
\begin{aligned}
& H=i \omega_{1} q_{1}^{\prime \prime} p_{1}^{\prime \prime}+i \omega_{2} q_{2}^{\prime \prime} p_{2}^{\prime \prime}+ \\
& \sum_{\alpha+\delta=3}^{\infty} h_{\alpha_{1}, \alpha_{2} \delta_{1}, \delta_{2}}^{\prime} q_{1}^{\prime \alpha_{1}} q_{2}^{\prime \prime \alpha_{2}} p_{1}^{\prime \prime \delta_{1}} p_{2}^{\prime \prime \delta_{2}}
\end{aligned}
$$

If $h_{\alpha_{1} \alpha_{2} \delta_{1} \delta_{2}}^{\prime}=x_{\alpha_{1} \alpha_{2} \delta_{1} \delta_{2}}+i y_{\alpha_{1} \alpha_{2} \delta_{1} \delta_{2}}$ then,

$$
\begin{aligned}
& x_{0030}=h_{0030}-\frac{1}{\omega_{1}^{2}} h_{2010} ; \quad y_{0030}=\frac{1}{\omega_{1}} h_{1020}-\frac{1}{\omega_{1}^{3}} h_{3000} ; \\
& x_{1020}=-\frac{1}{2} h_{1020}-\frac{3}{2 \omega_{1}^{2}} h_{3000} ; \quad y_{1020}=\frac{3 \omega_{1}}{2} h_{0030}+\frac{1}{2 \omega_{1}} h_{2010} ; \\
& x_{0120}=-\frac{\omega_{2}}{2} h_{0021}+\frac{1}{2 \omega_{1}} h_{1110}+\frac{\omega_{2}}{2 \omega_{1}^{2}} h_{2001} ; \\
& y_{0120}=-\frac{1}{2} h_{0120}-\frac{\omega_{2}}{2 \omega_{1}} h_{1011}+\frac{1}{2 \omega_{1}^{2}} h_{2100} ;
\end{aligned}
$$$$
x_{1011}=-\omega_{1} h_{0021}-\frac{1}{\omega_{1}} h_{2001} ; \quad y_{1011}=\frac{\omega_{1}}{\omega_{2}} h_{0120}+\frac{1}{\omega_{1} \omega_{2}} h_{2100} ;
$$$$
x_{1002}=-\frac{\omega_{1}}{2 \omega_{2}} h_{0111}-\frac{1}{2} h_{1002}+\frac{1}{2 \omega_{2}^{2}} h_{1200} ;
$$$$
y_{1002}=-\frac{\omega_{1}}{2} h_{0012}+\frac{\omega_{1}}{2 \omega_{2}^{2}} h_{0210}+\frac{1}{2 \omega_{2}^{2}} h_{1101}
$$$$
x_{0012}=-h_{0012}+\frac{1}{\omega_{2}^{2}} h_{0210}-\frac{1}{\omega_{1} \omega_{2}} h_{1101} ;
$$

$$
y_{0012}=\frac{1}{\omega_{2}} h_{0111}-\frac{1}{\omega_{1}} h_{1002}+\frac{1}{\omega_{1} \omega_{2}^{2}} h_{1200} \text {; }
$$


$x_{0111}=\frac{\omega_{2}}{\omega_{1}} h_{1002}+\frac{1}{\omega_{1} \omega_{2}} h_{1200} ; y_{0111}=-\omega_{2} h_{0012}-\frac{1}{\omega_{2}} h_{0210} ;$

$x_{0201}=\frac{-\omega_{2}}{4} h_{0102}-\frac{3}{4 \omega_{2}} h_{0300} ; y_{0201}=\frac{3 \omega_{2}^{2}}{4} h_{0003}+\frac{1}{4} h_{0201}$;

$x_{0003}=\frac{-1}{\omega_{2}} h_{0102}+\frac{1}{\omega_{2}^{3}} h_{0300}$;

$y_{0003}=-h_{0003}+\frac{1}{\omega_{2}^{2}} h_{0201} ;$

The other coefficients of third order terms are obtained by the formula:

$$
\begin{aligned}
& h_{\alpha_{1} \alpha_{2} \delta_{1} \delta_{2}}^{\prime}=\left(y_{\alpha_{1} \alpha_{2} \delta_{1} \delta_{2}}+i x_{\alpha_{1} \alpha_{2} \delta_{1} \delta_{2}}\right) \\
& \times\left(\frac{-\omega_{1}}{2}\right)^{\delta_{1}-\alpha_{1}}\left(\frac{\omega_{2}}{2}\right)^{\delta_{2}-\alpha_{2}}
\end{aligned}
$$

Using Birkhoff's transformation $\left(q_{j}^{\prime \prime}, p_{j}^{\prime \prime}\right) \rightarrow\left(q_{j}^{\prime \prime \prime}, p_{j}^{\prime \prime \prime}\right)$, all the third order terms from the Hamiltonian (6.10) is nullified provided the third order resonance does not occur. This transformation is introduced by means of the generating function which is given as follows:

$s=q_{1}{ }^{\prime \prime} p_{1}^{\prime \prime \prime}+q_{2}^{\prime \prime} p_{2}^{\prime \prime \prime}+s_{3}+s_{4} ;$

where, $q_{i}^{\prime \prime \prime}=q_{i}^{\prime \prime}+\frac{\partial s_{3}}{\partial p_{i}^{\prime \prime \prime}}+\frac{\partial s_{4}}{\partial p_{i}^{\prime \prime \prime}}$;

$p_{i}^{\prime \prime}=p_{i}^{\prime \prime \prime}+\frac{\partial s_{3}}{\partial q_{i}^{\prime \prime}}+\frac{\partial s_{4}}{\partial q_{i}^{\prime \prime}} ;(i=1,2)$

Using equation (6.4) and (6.6), expanding and equating the terms of the same degree on the two sides, we obtain:

$H_{2}^{\prime}\left(q_{1}^{\prime \prime}, q_{2}^{\prime \prime}, p_{1}^{\prime \prime \prime}, p_{2}^{\prime \prime \prime}\right)=H_{2}\left(\left(q_{1}^{\prime \prime}, q_{2}^{\prime \prime}, p_{1}^{\prime \prime \prime}, p_{2}^{\prime \prime \prime}\right)\right.$

$\sum_{i=1}^{2}\left[-\frac{\partial s_{3}}{\partial p_{i}^{\prime \prime \prime}} \frac{\partial H_{2}^{\prime}}{\partial p_{i}^{l^{\prime \prime}}}+\frac{\partial s_{3}}{\partial q_{i}^{\prime \prime}} \frac{\partial H_{2}}{\partial p_{i}^{\prime \prime \prime}}\right]+$

$H_{3}\left(q_{1}^{\prime \prime}, q_{2}^{\prime \prime}, p_{1}^{\prime \prime \prime}, p_{2}^{\prime \prime \prime}\right)=H_{3}^{\prime}\left(q_{1}^{\prime \prime}, q_{2}^{\prime \prime}, p_{1}^{\prime \prime \prime}, p_{2}^{\prime \prime \prime}\right)=0$;

$\sum_{i=1}^{2}\left[-\frac{\partial s_{4}}{\partial p_{i}^{\prime \prime \prime}} \frac{\partial H_{2}^{\prime}}{\partial q_{i}^{\prime \prime}}+\frac{\partial s_{4}}{\partial q_{i}^{\prime \prime}} \frac{\partial H_{2}}{\partial p_{i}^{\prime \prime \prime}}\right]+K_{4}=0 ;$

$H_{4}^{\prime}=\sum_{i=1}^{2}\left[-\frac{\partial s_{3}}{\partial p_{i}^{\prime \prime}} \frac{\partial H_{3}^{\prime}}{\partial q_{i}^{\prime \prime}}+\frac{\partial s_{3}}{\partial q_{i}^{\prime \prime}} \frac{\partial H_{3}}{\partial p_{i}^{\prime \prime \prime}}\right]+H_{4}$

$-K_{4}$

where, $\mathrm{K}_{4}$ is the term other than the homogeneous ones in $\mathrm{q}_{1}, \mathrm{p}_{1}$ and $\mathrm{q}_{2}, \mathrm{p}_{2}$. In equation (6.14) the new variables $q_{1}^{\prime \prime \prime}$ and $p_{2}^{\prime \prime \prime}$ can be replaced by $q_{1}^{\prime \prime}$ and $p_{2}$ " by implicit function theorem.

Since our system is autonomous we have: $\frac{\partial s_{3}}{\partial t}=\frac{\partial s_{4}}{\partial t}=0$.

Again, if we put

$H_{3}=\sum_{\alpha+\delta=3}^{\infty} h_{\alpha_{1}, \alpha_{2} \delta_{1}, \delta_{2}} q_{1}^{\prime \prime \prime} \alpha_{1} q_{2}^{\prime \prime \prime} \alpha_{2} p_{1}^{\prime \prime \prime} \delta_{1} p_{2}{ }^{\prime \prime} \delta_{2} ; s_{3}=\sum_{\alpha+\delta=3}^{\infty} g_{\alpha_{1}, \alpha_{2} \delta_{1}, \delta_{2}} q_{1}^{\prime \prime \prime \prime} \alpha_{1} q_{2}{ }^{\prime \prime \prime} \alpha_{2} p_{1}^{\prime \prime \prime} \delta_{1} p_{2}{ }^{\prime \prime \prime} \delta_{2}$
Using (6.14), we get:

$g_{\alpha_{1}, \alpha_{2} \delta_{1}, \delta_{2}}=\frac{i h_{\alpha_{1}, \alpha_{2} \delta_{1}, \delta_{2}}^{\prime}}{\left(\alpha_{1}-\delta_{1}\right) \omega_{1}+\left(\alpha_{2}-\delta_{2}\right) \omega_{2}}$

With the help of the third equation of (6.14), the new Hamiltonian inclusive of the fourth order terms is given as:

$H^{\prime}=i \omega_{1} q_{1}^{\prime \prime \prime} p_{1}^{\prime \prime \prime}+i \omega_{2} q_{2}^{\prime \prime \prime} p_{2}^{\prime \prime \prime}-c_{20}\left(q_{1}^{\prime \prime \prime} p_{1}^{\prime \prime \prime \prime}\right)^{2}$

$+c_{11}\left(q_{1}^{\prime \prime \prime} p_{1}^{\prime \prime \prime}\right)\left(q_{2}^{\prime \prime \prime} p_{2}^{\prime \prime \prime}\right)-c_{02}\left(q_{2}^{\prime \prime \prime} p_{2}^{\prime \prime \prime}\right)^{2}+\ldots$

where,

$K_{4}=H_{4}\left(q_{1}^{\prime \prime \prime}, q_{2}^{\prime \prime \prime}, p_{1}^{\prime \prime \prime}, p_{2}^{\prime \prime \prime}\right)-h_{2020}^{\prime} q_{1}^{\prime \prime \prime 2} p_{1}^{\prime \prime \prime}$

$-h^{\prime}{ }_{1111}\left(q_{1}^{\prime \prime \prime} p_{1}^{\prime \prime \prime}\right)\left(q_{2}^{\prime \prime \prime} p_{2}^{\prime \prime \prime}\right)-h^{\prime}{ }_{0202} q_{2}^{\prime \prime \prime 2} p_{2}^{\prime \prime \prime 2}$;

$c_{20}=-h_{2020}^{\prime}-\frac{3 \omega_{1}^{2}}{8}\left(x_{0030}^{2}+y_{0030}^{2}\right)-$

$\frac{3}{2}\left(x_{1020}^{2}+y_{1020}^{2}\right)+\frac{1}{2}\left(x_{1011}^{2}+y_{1011}^{2}\right)$

$-\frac{\omega_{1}^{2}}{2 \omega_{2}\left(2 \omega_{1}-\omega_{2}\right)}\left(x_{0120}^{2}+y_{0120}^{2}\right)+$

$\frac{\omega_{1}^{2} \omega_{2}}{8\left(2 \omega_{1}+\omega_{2}\right)}\left(x_{0021}^{2}+y_{0021}^{2}\right)$

$c_{11}=h^{\prime}{ }_{1111}-\frac{2 \omega_{2}^{2}}{\omega_{1}\left(\omega_{1}-2 \omega_{2}\right)}\left(x_{1002}^{2}+y_{1002}^{2}\right)$

$+\frac{\omega_{1} \omega_{2}^{2}}{2\left(\omega_{1}+2 \omega_{2}\right)}\left(x_{0012}^{2}+y_{0012}^{2}\right)$

$-\frac{\omega_{2} \omega_{1}^{2}}{2\left(2 \omega_{1}+\omega_{2}\right)} \times\left(x_{0021}^{2}+y_{0021}^{2}\right)$

$-\frac{2 \omega_{1}^{2}}{\left(2 \omega_{1}-\omega_{2}\right) \omega_{2}}\left(x_{0120}^{2}+y_{0120}^{2}\right)$

$+2\left(x_{0111} x_{1020}+y_{0111} y_{1020}\right)$

$-\frac{4}{\omega_{2}}\left(x_{0201} y_{1011}+x_{1011} y_{0201} ;\right)$

$c_{02}=h_{0202}^{\prime}+\frac{3 \omega_{2}^{2}}{8}\left(x_{0003}^{2}+y_{0003}^{2}\right)+$

$\frac{6}{\omega_{2}^{2}}\left(x_{0201}^{2}+y_{0201}^{2}\right)-$

$\frac{\omega_{2}^{2}}{2 \omega_{1}\left(\omega_{1}-2 \omega_{2}\right)}\left(x_{1002}^{2}+y_{1002}^{2}\right)$

$-\frac{1}{2}\left(x_{0111}^{2}+y_{0111}^{2}\right)$

$-\frac{\omega_{1} \omega_{2}^{2}}{8\left(\omega_{1}+2 \omega_{2}\right)}\left(x_{0012}^{2}+y_{0012}^{2}\right)$;

$h_{2020}^{\prime}=-\frac{3}{2} \omega_{1}^{2} h_{0040}-\frac{3}{2 \omega_{1}^{2}} h_{4000}-\frac{1}{2} h_{2020}$;

$h_{1111}^{\prime}=\omega_{1} \omega_{2} h_{0022}+\frac{1}{\omega_{1} \omega_{2}} h_{2200}+\frac{\omega_{1}}{\omega_{2}} h_{0220}$

$+\frac{\omega_{2}}{\omega_{1}} h_{2002}$ 


$$
h_{0202}^{\prime}=\frac{-3}{2} \omega_{2}^{2} h_{0004}-\frac{3}{2 \omega_{2}^{2}} h_{0400}-\frac{1}{2} h_{0202}
$$

Now, KAM theorem is applied where the values of $D=c_{20} \omega_{2}^{2}+c_{11} \omega_{1} \omega_{2}+c_{02} \omega_{1}^{2} \neq 0$ is calculated. Tables 3 and 4 show different values of $D$ satisfying $\beta_{1}+\beta_{2}<1$ and $\beta_{1}+\beta_{2}>1$ respectively. Table 5 shows the values of $\mathrm{D}$ for the binary systems ( Achird, Luyten, $\alpha$ Cen- AB, Kruger 60, Xi Bootis) .It is found that $\mathrm{D} \neq 0$, in all the cases of radiation pressures taken in general and in binary systems in particular. (See Appendices for table)

\section{Discussion and conclusion}

The existence of resonance and stability of the Lagrangian triangular equilibrium points in ER3BP is investigated considering both the body as radiating; under the non-resonance case using KAM theory in circular as well as elliptical cases. This work is generalization of the work of [Narayan and Singh (2014)] by considering the third and fourth order resonance for different values of radiation pressures satisfying both the conditions

$\beta_{1}+\beta_{2}>1 ; \beta_{1}+\beta_{2}<1, \beta_{1}, \beta_{2}<1$. in general and binary systems in particular. It is noticed that the system experiences resonance at $\omega_{1}=2 \omega_{2}, \omega_{1}=3 \omega_{2}$ for different values of radiation pressures in circular and elliptical case. The case $\omega_{1}=\omega_{2}$ corresponds to the boundary region of the stability for the system both in circular and elliptical cases .It is observed that resonance of the third and fourth order exists in ER3BP under the radiating primaries for all values of $\mu, \beta_{1}, \beta_{2}$ taken.

Also it is found that in case of binary systems $\omega_{1}=\omega_{2}$ occurs since $\mu \geq 0.0385209$

$\begin{array}{llll}\text { It is clear from table } \quad \text { (4) } & \text { that } \\ \text { for } \quad \mu=0.025, \beta_{1}=0.1, \beta_{2}=0.5, D=-5803.5 \quad \text {; } & \text { and }\end{array}$ for $\mu=0.025, \beta_{1}=0.1, \beta_{2}=0.05, D=8846.7$ there is change in the sign of $\mathrm{D}$ for both circular and elliptical cases. This change in sign suggests that for $\mu=0.025$ and $\beta_{1}=0.1$ there exists $0.05 \leq \beta_{2} \leq 0.5$ such that $\mathrm{D}$ vanishes. Similarly, it is observed that for $\mu=0.025, \beta_{1}=0.1$, there exists $\beta_{2}$ such that $0.04 \leq \beta_{2} \leq 0.4$ for which $\mathrm{D}=0$ in both the circular and elliptical cases. It is noticed that, expect for the two cases discussed, KAM theorem is applicable, hence showing that the triangular equilibrium points are stable.

It is seen that the binary systems (Achird, Luyten, $\alpha$ Cen- AB, Kruger 60 , and $\mathrm{Xi}$ Bootis) are stable as $\mathrm{D} \neq 0$. It is also found that except for some cases for all values of the radiation pressures and for $\mu<0.0385209$ the triangular equilibrium points are stable.

\section{References}

[1] Ammar MK (2008), the effect of solar radiation pressure on the Lagrangian points in the elliptical restricted three body problem, Astrophysics \& Space Science, 313, 393-408. http://dx.doi.org/10.1007/s10509-007-9709-z.

[2] Arnold VI (1963a), Proof of A. N. Kolmogorov's theorem on the preservation of quasiperiodic motions under small perturbations of the Hamiltonian, Russian Math. Surveys, 18, 5, 9-36.

[3] Arnold VI (1963b), Small divisor problems in classical and celestial mechanics, Russian Mathematical Surveys 18(6), 85-192. http://dx.doi.org/10.1070/RM1963v018n06ABEH001143.

[4] Bennet A (1965), Characteristics exponents of the five equilibrium solutions in the elliptically restricted problem, Icarus 4, 177-187. http://dx.doi.org/10.1016/0019-1035(65)90060-6.

[5] Bhatnagar KB, Gupta U \& Bhadrawaj R (1994), Celestial Mechanics and Dynamical Astronomy, 59, 345-374. http://dx.doi.org/10.1007/BF00692102.
[6] Conxita P (1995), Ejection collision orbits with the more massive primary in the planar elliptic restricted three body problem, Celestial Mechanics and Dynamical Astronomy, 61, 315-331. http://dx.doi.org/10.1007/BF00049513.

[7] Danby, JMA (1964), Stability of the triangular points in the elliptic restricted problem of three bodies, Astronomical Journal, 69, 165-172. http://dx.doi.org/10.1086/109254.

[8] Erdi B (2009), A parametric study of stability and resonance around L4 in the elliptical restricted three body problem, Celestial Mechanics and Dynamical Astronomy, 104,145- 158 http://dx.doi.org/10.1007/s10569-009-9197-2.

[9] Grebenikov EA (1964), On the stability of the Lagrangian Triangular Solutions of the Restricted Elliptic Three Body problem, Soviet Astronomy, 8(3), 451-459.

[10] Gyorgyey J (1985), on the nonlinear stability of motions around the elliptical restricted problem of three bodies, Celestial Mechanics and Dynamical Astronomy, 36(3), 281-285. http://dx.doi.org/10.1007/BF01230741.

[11] Halan PP \& Rana N (2001), The Existence and stability of equilibrium points in the Robe's restricted three body problem, Celestial Mechanics and Dynamical Astronomy, 79, 145-155. http://dx.doi.org/10.1023/A:1011173320720.

[12] Kumar S \& Ishwar B (2011) Location of collinear equilibrium points in the generalized photogravitational elliptical restricted three body problem, Int. Journal of Sciience \& Technology, 3(2), 157-162.

[13]Kumar V \& Choudhary RK (1986), On the stability of the triangular libration points for the photogravitational circular restricted problem of three bodies when both of the attracting bodies are radiating as well, Celestial Mechanics, 40(2), 155-170. http://dx.doi.org/10.1007/BF01230257.

[14]Liapnuov AM (1956), the general problem of stability of Motion, Acad. Science USSR

[15]Manju. \& Choudhary RK (1985), on the stability of triangular libration points taking into account the light pressure for the circular restricted problem of three bodies, Celestial Mechanics, 36, 165.

[16] Markeev AP (1978), Libration points in celestial Mechanics and Cosmodynamics, nauka, Moscow, Russia.

[17] Markellos VV, Perdios E \& Labropoulou P (1992), Linear stability of the triangular equilibrium points in the phptogravitational elliptical restricted three body problem, Astrophysics \& Space Science, 194, $207-$ 213. http://dx.doi.org/10.1007/BF00643991.

[18] Markellos VV (1996), Nonlinear stability zones around triangular equilibria in the plane circular restricted three-body problem, Astro$\begin{array}{lllll}\text { physics } & \& & \text { Space } & \text { Science, } & 245\end{array}$ http://dx.doi.org/10.1007/BF00637811.

[19]Narayan, A \& Singh.N (2014a), Motion and stability of triangular equilibrium points in elliptical restricted three body problem under the radiating primaries, Astrophysics \& Space Science, 352 (1), 57-70. http://dx.doi.org/10.1007/s10509-014-1903-1.

[20]Narayan, A., Singh.N. (2014c), Resonance stability of triangular equilibrium points in elliptical restricted three body problem under the radiating primaries, Astrophysics \& Space Science, 353 (2) 441-455. http://dx.doi.org/10.1007/s10509-014-2085-6.

[21] Narayan, A \& Singh N (2014b), Stability of triangular lagrangian points in elliptical restricted three body problem under the radiating binary systems, Astrophysics \& Space Science, 353 (2) 457-464. http://dx.doi.org/10.1007/s10509-014-2014-8.

[22] Roberts G (2002), linear stability of the elliptic Lagrangian triangle solution in the three body problem, Differential equation, 182, 191218. http://dx.doi.org/10.1006/jdeq.2001.4089.

[23] Sahoo SK \& Ishwar B (2000), Stability of collinear equilibrium points in the generalized photo gravitational elliptic restricted three-body problem, Bulletin Astronomical Society India, 28, 579-586.

[24]Selaru D \& Cucu-Dumitrescu C (1995), Infinitesimal orbit around Lagrange points in the elliptic restricted three body problem, Celestial Mechanics and Dynamical Astronomy, 61(4), 333- 346. http://dx.doi.org/10.1007/BF00049514.

[25] Singh J \& Umar A (2012a), Motion in the photogravitational elliptic restricted three-body problem under an oblate primary, Astronomical Journal, 143, 109. http://dx.doi.org/10.1088/0004-6256/143/5/109.

[26] Singh J \& Umar A, (2012b), on the stability of triangular equilibrium points in the elliptic R3BP under radiating and oblate primaries, As$\begin{array}{lllll}\text { trophysics } \& \quad \text { Space } & \text { Science, } 341, & 349 .\end{array}$ http://dx.doi.org/10.1007/s10509-012-1109-3.

[27] Szebebely V (1967), Stability of the points of equilibrium in the restricted problem, Astronomical Journal, 72, 7-9. http://dx.doi.org/10.1086/110195.

[28] Usha T, Narayan A \& Ishwar B (2014), Effects of Radiation and Triaxiality of primaries on triangular equilibrium points in elliptic re- 
stricted three body problem, Astrophysics \& Space Science, 349, 151164. http://dx.doi.org/10.1007/s10509-013-1655-3.

[29]Zimvoschikov AS \& Thakai VN (2004), Instability of libration points and resonance phenomena in the photogravitational in the elliptical restricted three body problem, Solar System Research, 38 (2), 155-163. http://dx.doi.org/10.1023/B:SOLS.0000022826.31475.a7.

\section{Appendices:}

Table 1: Values of $\mu$ at $\omega_{1}=\omega_{2}, \omega_{1}=2 \omega_{2}, \omega_{1}=3 \omega_{2}$ when $\beta_{1}+\beta_{2}>1$.

\begin{tabular}{|c|c|c|c|c|c|c|c|}
\hline \multirow{2}{*}{$\beta_{1}$} & \multirow{2}{*}{$\beta_{2}$} & \multicolumn{3}{|l|}{$\mathrm{e}=0$} & \multicolumn{3}{|c|}{$\mathrm{e}=0.0489$} \\
\hline & & $\mu_{01}$ & $\mu_{02}$ & $\mu_{03}$ & $\mu_{01 \mathrm{e}}$ & $\mu_{02 \mathrm{e}}$ & $\mu_{03 \mathrm{e}}$ \\
\hline 0.87 & 0.29 & 0.0304 & 0.0192 & 0.0107 & 0.0282 & 0.0181 & 0.0055 \\
\hline 0.87 & 0.34 & 0.0301 & 0.0190 & 0.0106 & 0.0277 & 0.0178 & 0.0052 \\
\hline 0.87 & 0.54 & 0.0290 & 0.0184 & 0.0103 & 0.0259 & 0.0167 & 0.0038 \\
\hline 0.87 & 0.59 & 0.0288 & 0.0182 & 0.0102 & 0.0255 & 0.0165 & 0.0034 \\
\hline 0.92 & 0.19 & 0.0306 & 0.0194 & 0.0108 & 0.0286 & 0.0183 & 0.0059 \\
\hline 0.92 & 0.24 & 0.0304 & 0.0192 & 0.0107 & 0.0282 & .0181 & 0.0055 \\
\hline 0.92 & 0.64 & 0.0283 & 0.0194 & 0.0108 & 0.0246 & 0.0159 & 0.0027 \\
\hline 0.82 & 0.39 & 0.0301 & 0.190 & 0.0106 & 0.0277 & 0.0178 & 0.0052 \\
\hline 0.82 & 0.49 & 0.0296 & 0.0187 & 0.0104 & 0.0268 & 0.0173 & 0.0045 \\
\hline 0.82 & 0.69 & 0.0285 & 0.0181 & 0.0101 & 0.0251 & 0.0162 & 0.0031 \\
\hline 0.82 & 0.79 & 0.0281 & 0.0178 & 0.0099 & 0.0242 & 0.0157 & 0.0024 \\
\hline 0.92 & 0.9 & 0.0312 & 0.0197 & 0.011 & 0.0223 & 0.0148 & 0.0009 \\
\hline 0.92 & 0.19 & 0.0306 & 0.0194 & 0.0108 & 0.0286 & 0.0183 & 0.0059 \\
\hline 0.92 & 0.79 & 0.0276 & 0.0175 & 0.0098 & 0.0233 & 0.0151 & 0.0017 \\
\hline 0.92 & 0.89 & 0.0272 & 0.0172 & 0.0096 & 0.0224 & 0.0146 & 0.0010 \\
\hline 0.72 & 0.49 & 0.0301 & 0.0190 & 0.0106 & 0.0277 & 0.0178 & 0.0052 \\
\hline 0.72 & 0.59 & 0.0296 & 0.0187 & 0.0104 & 0.0268 & 0.0173 & 0.0045 \\
\hline 0.72 & 0.89 & 0.0281 & 0.0178 & 0.0099 & 0.0242 & 0.0157 & 0.0024 \\
\hline 0.72 & 0.99 & 0.0276 & 0.0175 & 0.0098 & 0.0233 & 0.0151 & 0.0017 \\
\hline 0.82 & 0.39 & 0.0301 & 0.0190 & 0.0106 & 0.0277 & 0.0178 & 0.0052 \\
\hline 0.82 & 0.89 & 0.0276 & 0.0175 & 0.0098 & 0.0233 & 0.0151 & 0.0017 \\
\hline 0.82 & 0.99 & 0.0272 & 0.0172 & 0.0096 & 0.0224 & 0.0146 & 0.0010 \\
\hline 0.92 & 0.09 & 0.0312 & 0.0197 & 0.0110 & 0.0295 & 0.0189 & 0.0065 \\
\hline 0.92 & 0.19 & 0.0301 & 0.0190 & 0.0106 & 0.0268 & 0.0183 & 0.0059 \\
\hline 0.70 & 0.40 & 0.0307 & 0.0194 & 0.0108 & 0.0287 & 0.0184 & 0.0059 \\
\hline 0.70 & 0.50 & 0.0301 & 0.0191 & 0.0106 & 0.0278 & 0.0179 & 0.0052 \\
\hline 0.90 & 0.09 & 0.0313 & 0.0198 & 0.0111 & 0.0297 & 0.0190 & 0.0067 \\
\hline 0.90 & 0.19 & 0.0308 & 0.0195 & 0.0109 & 0.0288 & 0.0184 & 0.0060 \\
\hline 0.30 & 0.80 & 0.0307 & 0.0194 & 0.0108 & 0.0287 & 0.0184 & 0.0059 \\
\hline 0.30 & 0.90 & 0.0301 & 0.0191 & 0.0106 & 0.0278 & 0.0179 & 0.0052 \\
\hline 0.30 & 0.80 & 0.0307 & 0.0194 & 0.0100 & 0.0287 & 0.0184 & 0.0059 \\
\hline 0.50 & 0.60 & 0.0307 & 0.0194 & 0.0108 & 0.0287 & 0.0184 & 0.0059 \\
\hline 0.50 & 0.70 & 0.0301 & 0.0191 & 0.0106 & 0.0278 & 0.0179 & 0.0052 \\
\hline 0.60 & 0.50 & 0.0296 & 0.0187 & 0.0105 & 0.0287 & 0.0184 & 0.0059 \\
\hline 0.60 & 0.60 & 0.0301 & 0.0191 & 0.0106 & 0.0278 & 0.0179 & 0.0052 \\
\hline 0.70 & 0.40 & 0.0307 & 0.0194 & 0.0108 & 0.0287 & 0.0184 & 0.0059 \\
\hline 0.70 & 0.50 & 0.0301 & 0.0191 & 0.0106 & 0.0278 & 0.0179 & 0.0052 \\
\hline 0.20 & 0.70 & 0.0319 & 0.0202 & 0.0112 & 0.0260 & 0.0168 & 0.0038 \\
\hline 0.20 & 0.81 & 0.0313 & 0.0198 & 0.0110 & 0.0295 & 0.0189 & 0.0065 \\
\hline 0.80 & 0.80 & 0.0281 & 0.0178 & 0.0099 & 0.0243 & 0.0157 & 0.0025 \\
\hline 0.80 & 0.90 & 0.0276 & 0.0175 & 0.0098 & 0.0234 & 0.0152 & 0.0018 \\
\hline 0.90 & 0.70 & 0.0281 & 0.0178 & 0.0099 & 0.0243 & 0.0157 & 0.0025 \\
\hline 0.90 & 0.80 & 0.0276 & 0.0175 & 0.0098 & 0.0234 & 0.0152 & 0.0018 \\
\hline
\end{tabular}

\begin{tabular}{|c|c|c|c|c|c|c|c|}
\hline \multirow[b]{2}{*}{$\beta_{1}$} & \multirow[b]{2}{*}{$\beta_{2}$} & \multicolumn{2}{|l|}{$\mathrm{e}=0$} & \multicolumn{4}{|c|}{$\mathrm{e}=0.0489$} \\
\hline & & $\mu_{01}$ & $\mu_{02}$ & $\mu_{03}$ & $\mu_{01 \mathrm{e}}$ & $\mu_{02 \mathrm{e}}$ & $\mu_{03 \mathrm{e}}$ \\
\hline \multirow{2}{*}{0.01} & \multirow{2}{*}{0} & 0.03843 & 0.024238 & 0.01348 & 0.038 & 0.024 & 0.013 \\
\hline & & 1 & 6 & 5 & 4 & 2 & 4 \\
\hline \multirow{2}{*}{0.01} & 0.0 & 0.03834 & 0.024183 & 0.01345 & 0.038 & 0.024 & 0.013 \\
\hline & 1 & 3 & 6 & 5 & 3 & 2 & 4 \\
\hline \multirow{2}{*}{0.01} & 0.0 & 0.03825 & 0.024128 & 0.01342 & 0.038 & 0.024 & 0.013 \\
\hline & 2 & 5 & 9 & 5 & 3 & 1 & 3 \\
\hline \multirow{2}{*}{0.01} & 0.0 & 0.03816 & 0.024074 & 0.01339 & 0.038 & 0.024 & 0.013 \\
\hline & 3 & 7 & 4 & 5 & 2 & 1 & 2 \\
\hline \multirow{2}{*}{0.01} & 0.0 & 0.03808 & 0.024020 & 0.01336 & 0.038 & 0.024 & 0.013 \\
\hline & 4 & 0 & 1 & 5 & 1 & 0 & 2 \\
\hline \multirow{2}{*}{0.01} & 0.0 & 0.03799 & 0.023966 & 0.01333 & 0.038 & 0.024 & 0.013 \\
\hline & 5 & 3 & 1 & 5 & 0 & 0 & 1 \\
\hline \multirow{2}{*}{0.01} & 0.0 & 0.03790 & 0.029124 & 0.01333 & 0.037 & 0.023 & 0.013 \\
\hline & 6 & 6 & 3 & 0 & 9 & 9 & 0 \\
\hline \multirow{2}{*}{0.01} & 0.0 & 0.03782 & 0.023858 & 0.01327 & 0.037 & 0.023 & 0.013 \\
\hline & 7 & 0 & 9 & 0 & 8 & 9 & 0 \\
\hline \multirow{2}{*}{0.01} & 0.0 & 0.03773 & 0.023805 & 0.01324 & 0.037 & 0.023 & 0.012 \\
\hline & 8 & 4 & 6 & 7 & 7 & 8 & 9 \\
\hline 0.00 & 0.0 & 0.03842 & 0.024233 & 0.01348 & 0.038 & 0.024 & 0.013 \\
\hline 1 & 1 & 3 & 1 & 2 & 4 & 2 & 4 \\
\hline 0.00 & 0.0 & 0.03833 & 0.024178 & 0.01345 & 0.038 & 0.024 & 0.013 \\
\hline 1 & 2 & 4 & 1 & 2 & 3 & 2 & 4 \\
\hline 0.00 & 0.0 & 0.03842 & 0.024123 & 0.01342 & 0.038 & 0.024 & 0.013 \\
\hline 1 & 3 & 6 & 4 & 2 & 2 & 1 & 3 \\
\hline 0.00 & 0.0 & 0.03815 & 0.024069 & 0.01339 & 0.038 & 0.024 & 0.013 \\
\hline 1 & 4 & 8 & 0 & 2 & 2 & 1 & 2 \\
\hline 0.00 & 0.0 & 0.03807 & 0.024010 & 0.01336 & 0.038 & 0.024 & 0.013 \\
\hline 1 & 5 & 1 & 1 & 2 & 1 & 0 & 2 \\
\hline 0.00 & 0.0 & 0.03798 & 0.023960 & 0.13332 & 0.038 & 0.024 & 0.013 \\
\hline
\end{tabular}

\begin{tabular}{llllllll}
1 & 6 & 4 & 8 & 7 & 0 & 0 & 1 \\
0.00 & 0.0 & 0.03789 & 0.023907 & 0.01330 & 0.037 & 0.023 & 0.013 \\
1 & 7 & 8 & 0 & 3 & 9 & 9 & 0 \\
0.00 & 0.0 & 0.03781 & 0.023853 & 0.01327 & 0.037 & 0.023 & 0.013 \\
1 & 8 & 1 & 5 & 3 & 8 & 9 & 0 \\
0.1 & 0.0 & 0.03756 & 0.023699 & 0.01318 & 0.037 & 0.023 & 0.012 \\
& 1 & 4 & 0 & 9 & 5 & 7 & 8 \\
0.1 & 0.0 & 0.03747 & 0.023647 & 0.01316 & 0.037 & 0.023 & 0.012 \\
& 2 & 9 & 2 & 0 & 5 & 7 & 7 \\
0.1 & 0.0 & 0.03739 & 0.023594 & 0.01313 & 0.037 & 0.023 & 0.012 \\
& 3 & 5 & 8 & 1 & 4 & 6 & 6 \\
0.1 & 0.0 & 0.03731 & 0.023542 & 0.01310 & 0.037 & 0.023 & 0.012 \\
& 4 & 1 & 7 & 2 & 3 & 5 & 6 \\
0.1 & 0.0 & 0.03722 & 0.023490 & 0.01307 & 0.037 & 0.023 & 0.012 \\
& 5 & 8 & 8 & 4 & 2 & 5 & 5 \\
0.1 & 0.0 & 0.03714 & 0.023439 & 0.01304 & 0.037 & 0.023 & 0.012 \\
& 6 & 5 & 2 & 5 & 1 & 4 & 4 \\
0.1 & 0.0 & 0.03706 & 0.023387 & 0.01301 & 0.037 & 0.023 & 0.012 \\
& 7 & 2 & 8 & 7 & 0 & 4 & 3 \\
0.1 & 0.0 & 0.03698 & 0.023336 & 0.01298 & 0.036 & 0.023 & 0.012 \\
& 8 & 0 & 6 & 9 & 9 & 3 & 3 \\
\hline
\end{tabular}

Table 3: Values of $D$ when $\beta_{1}+\beta_{2}>1$

\begin{tabular}{|c|c|c|c|c|}
\hline$\mu$ & $\beta_{1}$ & $\beta_{2}$ & Values of $\mathrm{D}$ at $\mathrm{e}=0.0489$ & Values of $\mathrm{D}$ at $\mathrm{e}=0$ \\
\hline 0.001 & 0.87 & 0.29 & $-1.9573 \times 10^{7}$ & $-1.7809 \times 10^{7}$ \\
\hline 0.001 & 0.87 & 0.34 & $-1.8077 \times 10^{7}$ & $-1.6448 \times 10^{7}$ \\
\hline 0.001 & 0.87 & 0.54 & $-1.3725 \times 10^{7}$ & $-1.2489 \times 10^{7}$ \\
\hline 0.001 & 0.87 & 0.59 & $-1.2980 \times 10^{7}$ & $-1.1812 \times 10^{7}$ \\
\hline 0.001 & 0.92 & 0.19 & $-2.3668 \times 10^{7}$ & $-2.1534 \times 10^{7}$ \\
\hline 0.001 & 0.92 & 0.24 & $-2.1707 \times 10^{7}$ & $-1.9750 \times 10^{7}$ \\
\hline 0.001 & 0.92 & 0.59 & $-1.3136 \times 10^{7}$ & $-1.1953 \times 10^{7}$ \\
\hline 0.001 & 0.92 & 0.64 & $-1.2480 \times 10^{7}$ & $-1.1357 \times 10^{7}$ \\
\hline 0.005 & 0.82 & 0.39 & $-1.8242 \times 10^{5}$ & $--1.3089 \times 10^{5}$ \\
\hline 0.005 & 0.82 & 0.49 & $-1.6578 \times 10^{5}$ & $-1.5125 \times 10^{5}$ \\
\hline 0.005 & 0.82 & 0.69 & $-1.4942 \times 10^{5}$ & $-1.3658 \times 10^{5}$ \\
\hline 0.005 & 0.82 & 0.79 & $-1.4890 \times 10^{5}$ & $-1.3626 \times 10^{5}$ \\
\hline 0.005 & 0.92 & 0.09 & $-1.5074 \times 10^{5}$ & $-2.6245 \times 10^{5}$ \\
\hline 0.005 & 0.92 & 0.19 & $-2.4571 \times 10^{5}$ & $-2.2368 \times 10^{5}$ \\
\hline 0.005 & 0.92 & 0.79 & $-1.4705 \times 10^{5}$ & $-1.3442 \times 10^{5}$ \\
\hline 0.005 & 0.92 & 0.89 & $-1.5015 \times 10^{5}$ & $-1.3737 \times 10^{5}$ \\
\hline 0.010 & 0.72 & 0.49 & $-3.4213 \times 10^{5}$ & $-1.4939 \times 10^{5}$ \\
\hline 0.010 & 0.72 & 0.59 & $-3.3800 \times 10^{4}$ & $-1.4107 \times 10^{4}$ \\
\hline 0.010 & 0.72 & 0.89 & $-3.9107 \times 10^{4}$ & $-3.6163 \times 10^{4}$ \\
\hline 0.010 & 0.72 & 0.99 & $-4.3317 \times 10^{4}$ & $-4.0109 \times 10^{4}$ \\
\hline 0.010 & 0.82 & 0.39 & $-3.7221 \times 10^{4}$ & $-3.2987 \times 10^{4}$ \\
\hline 0.010 & 0.82 & 0.89 & $-3.7221 \times 10^{4}$ & $-3.4341 \times 10^{4}$ \\
\hline 0.010 & 0.82 & 0.99 & $-4.0951 \times 10^{4}$ & $-3.7835 \times 10^{4}$ \\
\hline 0.010 & 0.92 & 0.09 & $-5.2180 \times 10^{4}$ & $-4.7519 \times 10^{4}$ \\
\hline 0.015 & 0.70 & 0.40 & $-2.0233 \times 10^{4}$ & $-1.8562 \times 10^{4}$ \\
\hline 0.015 & 0.70 & 0.50 & $-2.1860 \times 10^{4}$ & $-1.8523 \times 10^{4}$ \\
\hline 0.015 & 0.80 & 0.30 & $-2.1816 \times 10^{4}$ & $-1.9943 \times 10^{4}$ \\
\hline 0.015 & 0.90 & 0.19 & $-2.5133 \times 10^{4}$ & $-2.2915 \times 10^{4}$ \\
\hline 0.015 & 0.30 & 0.80 & $-2.7543 \times 10^{4}$ & $-2.5753 \times 10^{4}$ \\
\hline 0.015 & 0.30 & 0.90 & $-3.1119 \times 10^{4}$ & $-2.9130 \times 10^{4}$ \\
\hline 0.015 & 0.30 & 0.80 & $-2.7543 \times 10^{4}$ & $-2.5753 \times 10^{4}$ \\
\hline 0.025 & 0.50 & 0.60 & $-3.8796 \times 10^{3}$ & $-3.7364 \times 10^{3}$ \\
\hline 0.025 & 0.50 & 0.70 & $-5.0133 \times 10^{3}$ & $-4.8078 \times 10^{3}$ \\
\hline 0.025 & 0.50 & 0.50 & $-2.2754 \times 10^{3}$ & $-2.7278 \times 10^{3}$ \\
\hline 0.025 & 0.60 & 0.60 & $-3.2053 \times 10^{3}$ & $-3.0871 \times 10^{3}$ \\
\hline 0.025 & 0.70 & 0.40 & $-1.0171 \times 10^{3}$ & $-1.0183 \times 10^{3}$ \\
\hline 0.025 & 0.70 & 0.50 & $-1.8149 \times 10^{3}$ & $-1.7656 \times 10^{3}$ \\
\hline 0.025 & 0.70 & 0.70 & $-3.4763 \times 10^{3}$ & $-3.3318 \times 10^{3}$ \\
\hline 0.025 & 0.70 & 0.80 & $-4.4176 \times 10^{3}$ & $-4.2228 \times 10^{3}$ \\
\hline 0.025 & 0.80 & 0.80 & $-3.6712 \times 10^{3}$ & $-3.5071 \times 10^{3}$ \\
\hline 0.030 & 0.50 & 0.60 & $-4.100 \times 10^{3}$ & $-3.9019 \times 10^{3}$ \\
\hline 0.030 & 0.50 & 0.70 & $-4.9224 \times 10^{3}$ & $-4.6827 \times 10^{3}$ \\
\hline 0.030 & 0.50 & 0.80 & $-5.8530 \times 10^{3}$ & $-5.5678 \times 10^{3}+64.951 \mathrm{i}$ \\
\hline 0.030 & 0.50 & 0.90 & $-6.8962 \times 10^{3}$ & $-6.5618 \times 10^{3}+83.781 \mathrm{i}$ \\
\hline 0.030 & 0.60 & 0.70 & $-4.2184 \times 10^{3}$ & $-4.008 \times 10^{3}+80.513 i$ \\
\hline 0.035 & 0.40 & 0.80 & $-5.8297 \times 10^{3}$ & $-5.5488 \times 10^{3}+1.064 \times 10^{2} \mathrm{i}$ \\
\hline 0.035 & 0.40 & 0.70 & $-4.9641 \times 10^{3}$ & $-4.7236 \times 10^{3}+1.275 \times 10^{2} \mathrm{i}$ \\
\hline 0.035 & 0.40 & 0.80 & $-5.829 \times 10^{3}$ & $-5.5488 \times 10^{3}+1.1064 \times 10^{2} \mathrm{i}$ \\
\hline 0.035 & 0.80 & 0.90 & $-6.8488 \times 10^{3}$ & $-3.3612 \times 10^{3}+1.2392 \times 10^{3} \mathrm{i}$ \\
\hline
\end{tabular}


Table 4: Values of D when $\beta_{1}+\beta_{2}<1$

\begin{tabular}{lllll}
\hline$\mu$ & $\beta_{1}$ & $\beta_{2}$ & Values of D at $\mathrm{e}=0.0489$ & Values of D at $\mathrm{e}=0$ \\
\hline 0.001 & 0.01 & 0.02 & $-2.2480 \times 10^{7}$ & $-2.0457 \times 10^{7}$ \\
0.001 & 0.01 & 0.03 & $-2.2083 \times 10^{7}$ & $-2.0097 \times 10^{7}$ \\
0.001 & 0.01 & 0.04 & $-2.1696 \times 10^{7}$ & $-1.9744 \times 10^{7}$ \\
0.001 & 0.01 & 0.05 & $-2.1317 \times 10^{7}$ & $-1.9400 \times 10^{7}$ \\
0.005 & 0.01 & 0.002 & $-2.4321 \times 10^{5}$ & $-2.2218 \times 10^{7}$ \\
0.005 & 0.01 & 0.003 & $-2.4321 \times 10^{5}$ & $-2.2187 \times 10^{7}$ \\
0.005 & 0.01 & 0.004 & $-2.4253 \times 10^{5}$ & $-2.2156 \times 10^{7}$ \\
0.005 & 0.01 & 0.005 & $-2.4218 \times 10^{5}$ & $-2.2125 \times 10^{7}$ \\
0.010 & 0.001 & 0.002 & $-2.4269 \times 10^{5}$ & $-4.2750 \times 10^{4}$ \\
0.010 & 0.001 & 0.003 & $-2.4235 \times 10^{5}$ & $-4.2715 \times 10^{4}$ \\
0.010 & 0.001 & 0.004 & $-2.4200 \times 10^{5}$ & $-4.2680 \times 10^{4}$ \\
0.010 & 0.001 & 0.005 & $-2.4166 \times 10^{5}$ & $-4.2645 \times 10^{4}$ \\
0.015 & 0.10 & 0.20 & $-2.0880 \times 10^{4}$ & $-1.9354 \times 10^{4}$ \\
0.015 & 0.10 & 0.30 & $-2.1141 \times 10^{4}$ & $-1.9657 \times 10^{4}$ \\
0.015 & 0.10 & 0.40 & $-2.1961 \times 10^{4}$ & $-2.0437 \times 10^{4}$ \\
0.015 & 0.10 & 0.50 & $-2.3345 \times 10^{4}$ & $-2.1777 \times 10^{4}$ \\
0.025 & 0.01 & 0.02 & $1.9584 \times 10^{4}$ & $1.7642 \times 10^{4}$ \\
0.025 & 0.01 & 0.03 & $1.7619 \times 10^{4}$ & $1.5852 \times 10^{4}$ \\
0.025 & 0.01 & 0.04 & $1.5882 \times 10^{4}$ & $1 . .4268 \times 10^{4}$ \\
0.025 & 0.01 & 0.05 & $1.4330 \times 10^{4}$ & $1.2856 \times 10^{4}$ \\
0.025 & 0.10 & 0.50 & $-5.8035 \times 10^{3}$ & $-6.4762 \times 10^{3}$ \\
0.025 & 0.10 & 0.05 & $8.8467 \times 10^{3}$ & $7.8920 \times 10^{3}$ \\
0.025 & 0.10 & 0.005 & $1.3043 \times 10^{3}$ & $1.1721 \times 10^{3}$ \\
0.025 & 0.10 & 0.40 & $-3.8410 \times 10^{3}$ & $-3.7766 \times 10^{3}$ \\
0.025 & 0.10 & 0.04 & $9.6539 \times 10^{3}$ & $8.6290 \times 10^{3}$ \\
0.030 & 0.10 & 0.50 & $-6.4125 \times 10^{3}$ & $-6.1125 \times 10^{3}$ \\
0.030 & 0.10 & 0.05 & $-2.1105 \times 10^{3}$ & $-2.0514 \times 10^{3}$ \\
0.030 & 0.10 & 0.40 & $-5.3322 \times 10^{3}$ & $-1.9878 \times 10^{3}$ \\
0.030 & 0.10 & 0.04 & $-2.0190 \times 10^{3}$ & $-2.5216 \times 10^{3}$ \\
0.035 & 0.10 & 0.04 & $-2.6500 \times 10^{3}$ & $-3.3861 \times 10^{3}$ \\
0.035 & 0.10 & 0.004 & $-2.4638 \times 10^{3}$ & $-2.4231 \times 10^{3}$ \\
0.035 & 0.10 & 0.20 & $-3.5610 \times 10^{3}$ & \\
0.035 & 0.10 & 0.02 & $-2.5459 \times 10^{3}$ & \\
\hline & & & & 30 \\
\hline
\end{tabular}

Table 5: Values of D for Binary Systems

\begin{tabular}{llllll}
\hline $\begin{array}{l}\text { Binaries } \\
\text { system }\end{array}$ & $\mu$ & $\beta_{1}$ & $\beta_{2}$ & eccentricity & Values of D \\
\hline Achird & 0.3949 & 0.0029 & 0.0003 & 0.4970 & $136.7-298.2 \mathrm{i}$ \\
$\begin{array}{l}\text { Luyten } \\
\alpha \text { Cen }\end{array}$ & 0.5 & 0.00002 & 0.000001 & 0.6200 & $9.4138-4.2464 \mathrm{i}$ \\
$\begin{array}{l}\mathrm{AB} \\
\text { Kruger- }\end{array}$ & 0.4519 & 0.0029 & 0.15 & 0.5179 & $-62.9098-3.8063 \mathrm{i}$ \\
$\begin{array}{l}60 \\
\text { Xi- }\end{array}$ & 0.3937 & 0.00008 & 0.00004 & 0.4100 & $-1311.6-2624 \mathrm{i}$ \\
bootis & 0.4231 & 0.0012 & 0.0012 & 0.5117 & $-99.4820-3.3311 \mathrm{i}$ \\
\hline
\end{tabular}

